Classification: Biological Science - Microbiology

\title{
Joint antibiotic and phage therapy: addressing the limitations of a seemingly ideal phage for treating Staphylococcus aureus infections
}

Brandon A. Berryhill' ${ }^{1}$, Douglas L. Huseby ${ }^{2}$, Ingrid C. McCall ${ }^{1}$, Diarmaid Hughes ${ }^{2}$, and Bruce R. Levin ${ }^{1 *}$

${ }^{1}$ Department of Biology, Emory University 1510 Clifton Rd NE, Atlanta, GA 30322, USA

${ }^{2}$ Department of Medical Biochemistry and Microbiology, Biomedical Center, Uppsala University, 751 23, Uppsala, Sweden.

*Corresponding Author: Bruce R. Levin, Emory University, 1510 Clifton Rd Atlanta, GA 30322, 404-727-2826, blevin@emory.edu. 


\begin{abstract}
In response to increasing frequencies of antibiotic-resistant pathogens, there has been a resurrection of interest in the use of bacteriophage to treat bacterial infections: phage therapy. Here we explore the potential of a seemingly ideal phage, $\mathrm{PYO}^{\mathrm{Sa}}$, for combination phage and antibiotic treatment of Staphylococcus aureus infections. (i) This K-like phage has a broad host range; all 83 tested clinical isolates of S.aureus tested were susceptible to $\mathrm{PYO}^{\mathrm{Sa}}$. (ii) Because of the mode of action of $\mathrm{PYO}^{\mathrm{Sa}} S$. aureus is unlikely to generate classical receptor-site mutants resistant to $\mathrm{PYO}^{\mathrm{Sa}}$; none were observed in the 13 clinical isolates tested. (iii) $\mathrm{PYO}^{\mathrm{Sa}}$ kills $S$. aureus at high rates. On the downside, the results of our experiments and tests of the joint action of $\mathrm{PYO}^{\mathrm{Sa}}$ and antibiotics raise issues that must be addressed before $\mathrm{PYO}^{\mathrm{Sa}}$ is employed clinically. Despite the maintenance of the phage, $\mathrm{PYO}^{\mathrm{Sa}}$ does not clear the populations of $S$. aureus. Due to the ascent of a phenotypically diverse array of small colony variants following an initial demise, the bacterial populations return to densities similar to that of phage-free controls. Using a combination of mathematical modeling and in vitro experiments, we postulate and present evidence for a mechanism to account for the demise-resurrection dynamics of $\mathrm{PYO}^{\mathrm{Sa}}$ and $S$. aureus. Critically for phage therapy, our experimental results suggest that treatment with $\mathrm{PYO}^{\mathrm{Sa}}$ followed by bactericidal antibiotics can clear populations of $S$. aureus more effectively than the antibiotics alone.
\end{abstract}

Keywords: Phage therapy; population dynamics; Staphylococcus aureus

\title{
Significance Statement
}

The increasing frequency of antibiotic-resistant pathogens has fostered a quest for alternative means to treat bacterial infections. Prominent in this quest is a therapy that predates antibiotics: bacteriophage. This study explores the potential of a phage, PYO ${ }^{\text {Sa }}$, for treating Staphylococcus aureus infections in combination with antibiotics. On first consideration, this phage, isolated from a commercial therapeutic cocktail, seems ideal for this purpose. The results of this population dynamic and genomic analysis study identify a potential liability of using PYO $^{\text {Sa }}$ for therapy. Due to the production of potentially pathogenic atypical small colony variants, PYOSa alone cannot eliminate $S$. aureus populations. However, we demonstrate that by following the administration of $\mathrm{PYO}^{\mathrm{Sa}}$ with bactericidal antibiotics, this limitation and potential liability can be addressed. 


\section{Introduction}

Driven by well-warranted concerns about the increasing frequencies of infections with antibiotic-resistant pathogens, there has been a resurrection of interest in, research on, and clinical trials with a therapy that predates antibiotics by more than 15 years: bacteriophage (1-4). One direction phage therapy research has taken is to engineer lytic (virulent) phages with properties that are anticipated to maximize their efficacy for treating bacterial infections in mammals (5-7). Primary among these properties are (i) a broad host range for the target bacterial species, (ii) mechanisms that prevent the generation of envelope or other kinds of high-fitness resistance in the target bacteria (8), (iii) the capacity to thwart the innate and adaptive immune systems of bacteria, respectively restriction-modification and CRISPR-Cas (7, 9, 10), (iv) the ability to survive, kill, and replicate on pathogenic bacteria colonizing or infecting mammalian hosts $(11,12)$ and $(v)$ little or no negative effects on the treated host (8).

To these five desired properties for therapeutic bacteriophage, we add a sixth: synergy with antibiotics. Phage-only treatment may be reasonable for compassionate therapy where the bacteria responsible for the infection are resistant to all available antibiotics (13-15). But from a practical perspective, for phage to become a widely employed mechanism for treating bacterial infections, they would have to be effective in combination with antibiotics. It would be unethical and unacceptable to clinicians and regulatory agencies to use phage independently for infections that can be effectively treated with existing antibiotics.

Although not specifically engineered for these properties, there is a Staphylococcal phage isolated from a therapeutic phage collection from the Eliava Institute in Tbilisi; we call PYOSa that on first consideration appears to have all six of the properties required to be an effective agent for therapy. (i) PYO ${ }^{\text {Sa }}$ is likely to have a broad host range for $S$, aureus. The receptor of this K-like Myoviridae is $\mathrm{N}$ acetylglucosamine in the wall-teichoic acid backbone of Staphylococcus aureus and is shared among most (16), if not all S. aureus; thereby suggesting PYO ${ }^{\text {Sa }}$ should be able to adsorb to and potentially replicate on and kill a vast number of clinical isolates of $S$. aureus. (ii) $S$. aureus does not generate classical, surface modification mutants resistant to PYOSa. Since the structure of the receptor of $\mathrm{PYO}^{\text {Sa }}$ is critical to the viability, replication, and virulence of these bacteria, the modifications in this receptor (17) may not be consistent with the viability or pathogenicity of $S$. aureus (18). (iii) The replication of $\mathrm{PYO}^{\mathrm{Sa}}$ is unlikely to be prevented by restrictionmodification (RM) or CRISPR-Cas. Despite a genome size of $127 \mathrm{~KB}$, the PYO ${ }^{\text {Sa }}$ phage has no GATC restriction sites for the $S$. aureus restriction enzyme Sau3A1 and only one restriction site, GGNCC, for the Sau961 restriction endonuclease $(19,20)$. There is no evidence for a functional CRISPR-Cas system in S. aureus or, to our knowledge, other mechanisms by which $S$. aureus may prevent the replication of this phage (21).

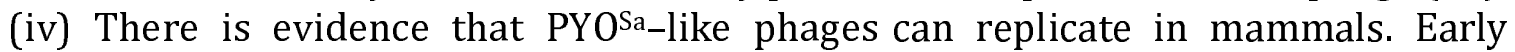
treatment with a phage with a different name but the same properties as $\mathrm{PYO}^{\mathrm{Sa}}$, 
Statuv, prevented mortality in otherwise lethal peritoneal infections of $S$. aureus in mice (22). A PYOSa-like phage has also been successfully used therapeutically in

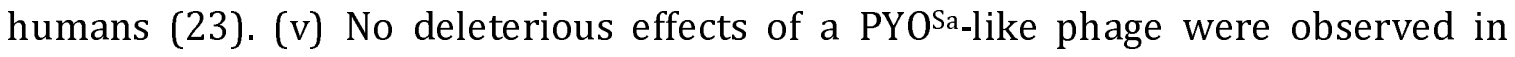
recent placebo-controlled trials with volunteers asymptotically colonized by $S$. aureus (19). (vi) Finally, there is evidence to suggest synergy with antibiotics. In vitro, PYOSa increased the efficacy of low concentrations of antibiotics for the treatment of biofilm populations of $S$. aureus (24).

With in vitro parameter estimation, population and evolutionary dynamic studies, and experiments with PYOSa and $S$. aureus Newman in combination with three different bacteriostatic and six different bactericidal antibiotics, we explore just how well PYOSa fits the above criteria for combination antibiotic and phage therapy. Our results suggest that $\mathrm{PYO}^{\mathrm{Sa}}$ scores well on most of these tests but does not get an " $\mathrm{A}$ ". As a consequence of selection for potentially pathogenic small colony variants, by itself $\mathrm{PYO}^{\text {Sa }}$ does not clear $S$. aureus infections; after an initial demise when confronted with this phage, although the phage continue to be present, the densities of the bacteria return to levels similar to those observed in the absence of this virus. By employing antibiotics and phage against $S$. aureus we could demonstrate synergy in clearing the bacterial population. However, there were significant differences in effectiveness depending on whether the antibiotics and phage were used simultaneously or in succession, and on whether the antibiotics used were bacteriostatic or bacteriocidal. Our most important result from a therapeutic perspective is that treatment of $S$. aureus cultures with PYOSa, followed by the administration of bactericidal antibiotics, is more effective at clearing the bacterial population than treatment with these antibiotics alone.

\section{Results}

(1) Bacteriophage PYOSa has a broad host range for S. aureus: We use two assays to determine the host range of $\mathrm{PYO}^{\mathrm{Sa}}$ : (i) the production of zones of inhibition in soft agar lawns and (ii) changes in the optical density of exponentially growing liquid cultures of $S$. aureus mixed with this phage. By both criteria, $S$. aureus Newman and 12 clinical isolates of methicillin-sensitive $S$. aureus from the Network on Antimicrobial Resistance (NARSA) collection (25) were all sensitive to $\mathrm{PYO}^{\mathrm{Sa}}$ and appeared to be unable to generate classically resistant mutants. Additional evidence for a broad host range of PYO ${ }^{\text {Sa }}$ comes from a survey of 71 clinical isolates of $S$. aureus, including 54 MRSA strains and phylogenetically similar species performed by LabCorp ${ }^{\mathrm{TM}}$ (SI Appendix, Table S1).

(2) $S$. aureus appears to be unable to generate classical, surface modification, mutants resistant to PYOSa: Evidence for this comes from experiments with $S$. aureus Newman and 12 clinical isolates from the NARSA collection (25). Single colonies of each strain were grown in the presence of $\sim 10^{6}$ phage particles per ml, and after 24 hours of exposure to the phage in liquid culture, the optical densities of exponentially growing $S$. aureus were no greater than that of the media without the bacteria, and by plating, we were unable to detect colonies from these cultures. 
(3) Bacterial population heterogeneity and the maintenance of phage: With the bacterial growth and phage infection parameters estimated for $S$. aureus Newman and PYOSa, a complete clearance of the bacteria in these experiments after less than 24 hours of exposure to $\mathrm{PYO}^{\mathrm{Sa}}$ is anticipated from a simple mass action model of the population dynamics of bacteria and phage (26). To determine whether this is the case empirically, we divided a culture with $\sim 10^{4}$ S. aureus Newman into 28 tubes. We let these cultures replicate for 2 hours, for an average density of $3 \times 10^{7}$ and then added $\sim 6 \times 10^{8} \mathrm{PYO}^{S a}$. At 24 hours, all 28 cultures were clear, and no bacterial colonies were found on the LB plate samplings of these cultures. However, by day seven, 26 of these 28 independent cultures were turbid or somewhat turbid. These turbid cultures all had phage at densities in excess of $5 \times 10^{6}$. Upon plating these 7 day cultures, colonies were detected with two distinct phenotypes: colonies similar in size to the ancestral wild-type $S$. aureus Newman and much smaller colonies, small colony variants (Fig. 1A).

To elucidate why PYOSa does not kill all of the $S$. aureus Newman in these liquid cultures, we prepared three independent $10 \mathrm{ml}$ cultures with $\sim 10^{8} \mathrm{~S}$. aureus Newman and $\sim 10^{7} \mathrm{PYO}^{\text {Sa }}$ and serially passaged these cultures for five days, transferring $1 / 100$ of these cultures to fresh media each day. The results of these serial transfer experiments are presented in Fig. 1B. As anticipated from the experiment with the 28 tube cultures, at 24 hours, all the flasks were clear. The densities of bacteria remaining in these cultures estimated by plating, $\sim 10^{3}$ per $\mathrm{ml}$, is probably an underestimate of the real density due to killing by phage on the plates. Additional support for the hypothesis that the densities of $S$. aureus in these liquid cultures is substantially greater than the CFU estimate is that the density of the PYO ${ }^{\text {Sa }}$ phage did not decline at each transfer, and therefore, the phage must have been replicating. This hypothesis was evaluated with a simple model (26) using the phage infection parameters estimated for PYO ${ }^{\text {Sa }}$ and S. aureus Newman. To maintain a population in a culture diluted by 100 -fold the phage density must increase by at least 100 -fold at each transfer and for this to occur, the product of the adsorption rated constant, $\delta$, the burst size $\beta$, and the density of sensitive bacteria, $N$, has to exceed 100 . With the estimated values, $\delta=4.8 \times 10^{-7}$ and $\beta=80$, the density of bacteria in these cultures would have to be at least $2.6 \times 10^{6}$ for the phage not to be diluted out.

Most intriguingly, in all three independent serial transfer populations with the PYOSa phage present, the CFU estimates of the density of $S$. aureus started to increase after the $2^{\text {nd }}$ transfer. By the $4^{\text {th }}$ and $5^{\text {th }}$ transfer, the density of cells in these cultures was similar to that of the phage-free controls, and the phage continued to be maintained, presumably because of the continued presence of phage-susceptible populations. To test this we spread bacterial aliquots onto agar and observed heterogeneity in colony growth with both fast-growing and small colony variants. Using a spot test we showed that cultures grown from fast-growing colonies, henceforth called the evolved bacteria, were susceptible to the original $\mathrm{PYO}^{\text {Sa }}$ as well as to the co-existing 
PYOSa. In contrast, the spot test showed that cultures grown from small colony variants isolated from these late transfer cultures were resistant to lysis by $\mathrm{PYO}^{\mathrm{Sa}}$.

To further evaluate the different bacterial phenotypes present in the late transfer cultures we serially passaged two small colony cultures in the presence of $\mathrm{PYO}^{\mathrm{Sa}}$ (Fig. 1C). We observed that cultures generated from small colony variants were unable to support the phage. These data, and the spot test data suggest an explanation for the maintenance of bacteria and phage throughout the original transfer experiment (Fig. 1B). Our hypothesis is that the bacterial population is polymorphic and includes sensitive cells capable of supporting phage growth as well as a non-sensitive population. We tested this hypothesis by mixing wild-type $S$. aureus Newman in equal frequency with small colony variants, and observed, consistent with the hypothesis, that the phage were maintained throughout the serial transfers and that the total bacterial population density remained similar to that in phage-free controls (Fig. 1D). As a separate test of the polymorphic population hypothesis, we made serial transfer experiments initiated with mixtures of PYO ${ }^{\text {Sa }}$ phage and evolved bacteria isolated from the $5^{\text {th }}$ transfer cultures (Fig. $1 E$ ). The results of these experiments are consistent with this reduced susceptibility hypothesis. Moreover, within a single transfer, the bacterial density returned to that of the phage-free controls and the phage declined, albeit at a rate less than that anticipated if they were not replicating at all, in two of the three cultures. In the two cultures where the phage declined significantly, the dominant bacterial population were small colony variants; while, the replicate with the co-existing phage was dominanted by bacteria that generated wild-type colony morphologies. See SI Appendix, Section II for a consideration of the between experiment variation in the population dynamics observed in Fig. $1 B$ and $1 E$.

A
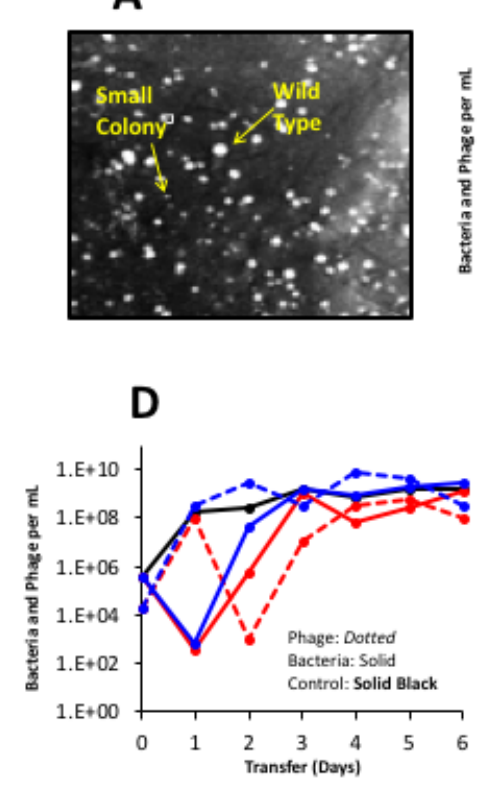

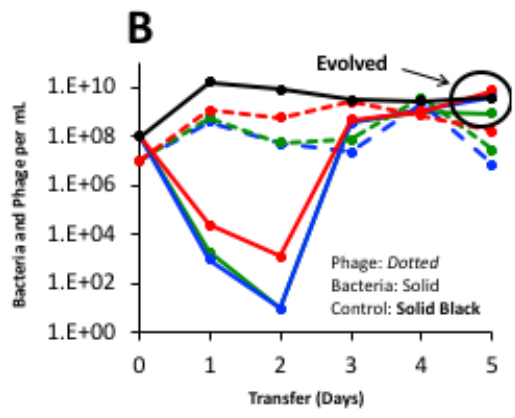

E

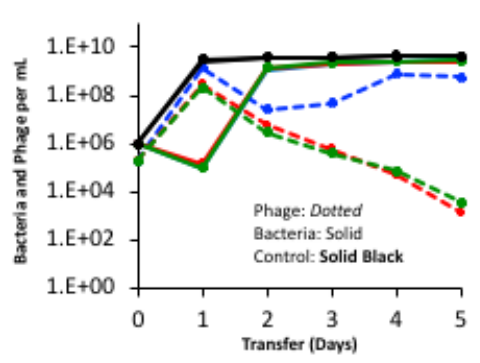

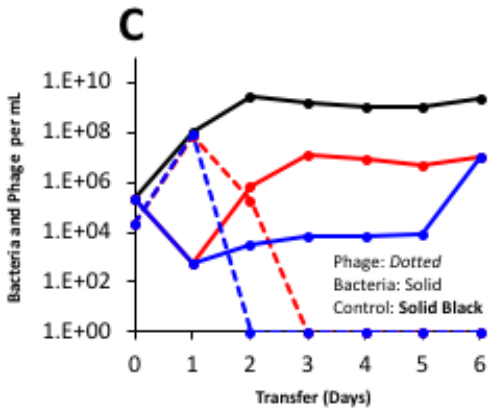

F

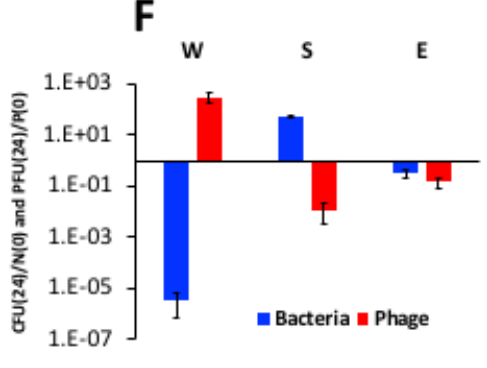


Fig. 1. Small colony variants and the population dynamics of PYO ${ }^{\text {Sa }}$ and $S$. aureus Newman in serial transfer culture. A. Wild type $S$. aureus and small colony variants visualized. B. Changes in densities of $S$. aureus Newman and PYO ${ }^{\text {Sa }}$ in three independent (red, green, blue) serial transfer experiments diluted 1/100 in fresh media daily. C. Changes in densities of $S$. aureus Newman and PYO ${ }^{\text {Sa }}$ in serial transfer cultures initiated two small colony variants isolated from the $5^{\text {th }}$ transfer of the cultures with $\mathrm{PYO}^{\mathrm{Sa}}$ and $S$. aureus Newman in panel B. D. Changes in densities of $S$. aureus Newman and $\mathrm{PYO}^{\mathrm{Sa}}$ in serial transfer cultures initiated with $\mathrm{PYO}^{\mathrm{Sa}}$ and equal densities of cultures derived from small colonies and the ancestral $S$. aureus Newman. E. Changes in densities of $S$. aureus and PYO ${ }^{\text {Sa }}$ in serial transfer cultures initiated with $\mathrm{PYO}^{\mathrm{Sa}}$ and single colonies of evolved (wild-type colony morphology) bacteria isolated from the $5^{\text {th }}$ transfer of the cultures in panel B. F. Changes in the ratio of bacteria and phage at time 0 and 24 hours. Wild type $S$. aureus Newman (W), small colony variants (S), and evolved bacteria (E). Three independent replicas.

Based on the preceding results and interpretations, we postulate that the bacteria are of three states, the ancestral wild-type, small colony variants, and what we call the evolved state, which have wild-type or near wild-type colony growth rate and rise to high densities after serial passaging in the presence of phage. Both the wildtype and the evolved are postulated to be capable of supporting phage growth whereas the small colonies type do not support phage growth.

(4) The genetic basis of bacterial colony growth rate variation: To determine the genetic basis of the differences between wild-type $S$. aureus Newman, the small colony variants, and evolved states, colonies with a range of sizes were isolated from early and late in serial passaging experiments similar to those shown in Fig. $1 B$. Whole genome sequencing of several clones revealed a clear sequence of events during the passaging. The first event was the selection of mutations in femA which encodes a protein responsible for assembling the pentaglycine interpeptide bridges in the S. aureus cell wall (SI Appendix, Table S2). These mutants all have a small colony variant phenotype. The range of observed colony sizes is representative of the diversity of mutations identified in $f e m A$, and presumably indicates a direct correlation between the growth rate and the severity of the defect in FemA function. We observed in later transfers larger colonies that carried a femA mutation and an additional mutation (see below) suggesting that this was a compensatory mutation restoring growth rate and creating the evolved state. The small colony variant phenotype of $S$. aureus is known to be very unstable and subject to rapid suppression by a wide variety of compensatory mutations (27). To investigate the nature of evolved state, we chose several different fem $A$ mutants and used these to select faster-growing colonies. Fast-growers were easily selected and were observed on agar as larger colonies growing above the slower-growing parental small colony strains. Whole genome sequencing revealed an array of suppressing mutations were selected, including an internal suppressor in femA, mutations in a variety of genes affecting secondary messenger metabolism, and frequent mutations in the transcriptional regulator sarA (SI Appendix, Table S3). We concluded that the initial event upon exposure of wild-type $S$. aureus Newman to $\mathrm{PYO}^{\text {Sa }}$ is the selection 
of small colony variants associated with mutations in femA. These slow-growing mutants then evolve to the faster-growing evolved state by the acquisition of suppressor mutations that frequently affect global transcriptional regulators.

(5) Phenotypic differences between wild-type, small colony, and evolved clones: The hypothesis above predicts that cultures derived from bacterial colonies of each state should respond differently when exposed to $\mathrm{PYO}^{\mathrm{Sa}}$. To test this, we co-cultured high densities of each bacterial state (W, S, and E) with PYO ${ }^{\text {Sa }}$ and determined the relative change in densities of both bacteria and phage, respectively $10^{8}$ and $10^{7}$ cells and particles per ml, after 24 hours exposure (Fig. 1F). Each of the three states exhibited a unique dynamic. The density of the wild-type and evolved bacteria declined, with the wild-type declining to a much greater extent than the evolved state. In contrast, the density of small colony variant bacteria increased. PYO ${ }^{\text {Sa }}$ phage increased on the wild-type bacteria but declined on both small colony variant and evolved state bacteria. The decline in phage was greater on small colony variants than on the evolved state.

Measurement of the growth rates in liquid culture of the three bacterial states confirmed the growth parameters previously inferred from colony sizes (SI Appendix, Table S4). Mutations causing an small colony phenotype on agar also caused severe growth defects in liquid culture, while the evolved clones with secondary suppressing mutations showed partial restoration of growth rate toward wild-type levels.

The pentapeptide crosslinks that FemA synthesizes are notably the target of lysostaphin. FemA mutants resistant to lysostaphin have previously been observed to become hypersensitive to penicillin antibiotics $(28,29)$. To test for evidence of these phenotypes the MIC of the three states were measured against lysostaphin and oxacillin (SI Appendix, Table S4). We observed that all of the strains carrying femA mutations (both small colony and evolved state) became resistant to lysostaphin and hypersensitive to oxacillin. Accordingly, the compensatory evolution from small colony variant to evolved state does not phenotypically recreate a wild-type phenotype: wild-type and evolved are genetically and phenotypically distinct states. Since FemA is responsible for adding the $2^{\text {nd }}$ and $3^{\text {rd }}$ glycines to the interpeptide bridge, while FemB is responsible for adding the $4^{\text {th }}$ and $5^{\text {th }}$ residues, mutations in $f e m A$ are expected to result in single glycine bridges between the cell wall peptides. To the degree that the femA mutants identified have different growth and susceptibility phenotypes, the cell wall defect in strains carrying these mutations is likely not complete, but rather represents a balance between full length pentapeptide crosslinks and single glycine crosslinks. FemA was recently reported to be essential (30) and it is notable that in our experiments no unequivocally fem $A$ null mutations were identified. Based on the number of mutants we have screened, this indicates that femA null mutants are either non-viable or are counterselected in these experiments. 
(6) $\mathrm{PYO}^{\text {Sa }}$ does not replicate on stationary phase S. aureus: In infected hosts, many of the bacteria will not be replicating, and thereby, phage would be particularly effective for treatment if they, like some antibiotics (31), could kill non-replicating $S$. aureus. To determine whether $\mathrm{PYO}^{\mathrm{Sa}}$ can kill and replicate on non-growing bacteria, three independent 48-hour stationary phase cultures were mixed with PYO $^{\text {Sa }}$ for average initial densities of $\sim 4 \times 10^{9}$ S. aureus and $10^{6} \mathrm{PYO} \mathrm{S}^{\mathrm{Sa}}$. The bacteria and phage were incubated with shaking for 24 hours, and the viable cell and phage densities were estimated and compared to the initial densities. There was no evidence for the stationary phase bacteria being killed, the mean and standard error of the $\mathrm{N}(24) / \mathrm{N}(0)$ ratio was $0.96 \pm 0.03$. Moreover and critically, there was a significant decline in the density of phage, $\mathrm{N}(24) / \mathrm{N}(0)=0.043 \pm 0.006$. Thus, not only does $\mathrm{PYO}^{\text {Sa }}$ not kill stationary phase $S$. aureus, these non-replicating bacteria act as a sink and could reduce the density of PYO ${ }^{\text {Sa }}$ from treated hosts.

The results of experiments to determine whether the decline in the density of phage can be attributed to changes in the medium, such as a high $\mathrm{pH}$, were negative. The density of PYO ${ }^{\text {Sa }}$ did not decline in sterile filtrates of 48-hour stationary phase cultures. There is, however, the suggestion that the bacteria must be viable to lead to the reduction in the viable density. When the $S$. aureus in the 48-hour stationary phase culture are killed with chloroform, the density of $\mathrm{PYO}^{\text {Sa }}$ does not decline

(7) Accounting for the population dynamics presented in Fig. 1B: In the SI Appendix, Section IV, Fig. S2, using a mathematical model and numerical solutions, we present a hypothesis for the kill-recovery dynamics observed for the bacteria, and the maintenance of the phage in the serial transfer cultures depicted in Fig. $1 B$. In accord with this hypothesis, (i) the bacterial population recovers from its initial demise due to predation by PYO ${ }^{\text {Sa }}$ phage, by the generation and ascent of small colony variants which are immune to, and selected by, the phage, and (ii) the phage are maintained because of the instability of the small colony variants which continously generate the evolved state bacteria upon which the phage can replicate.

(8) The joint action of PYOSa and antibiotics: To determine whether the action of antibiotics and $\mathrm{PYO}^{\mathrm{Sa}}$ would be synergistic or antagonistic in killing $S$ aureus Newman and the effect of these drugs on replication of PYO ${ }^{\mathrm{Sa}}$, we followed the change in the densities of bacteria and phage over 24 hours of exposure to antibiotics and phage in combination, and to phage alone. For these experiments, we mixed growing cultures of $S$. aureus Newman and PYO ${ }^{\text {Sa }}$ in MHII at a density of $4 \mathrm{X} 10^{6}$ with $4 \mathrm{X} 10^{5}$, respectively and super MIC concentrations of the antibiotics. The densities of bacteria and phage were estimated just before the antibiotics were added and 24 hours later, respectively $\mathrm{N}(0)$ and $\mathrm{N}(24)$, where $\mathrm{N}$ is either CFU or PFU. In these experiments, the antibiotics and phage were introduced into the growing cultures of $S$. aureus Newman in three different ways: (i) simultaneously, $\mathrm{AB}+\mathrm{PYO}^{\mathrm{Sa}}$, (ii) antibiotics first and phage 30 minutes later, $\mathrm{AB} \rightarrow \mathrm{PYO}^{\mathrm{Sa}}$, and (iii) phage first and antibiotics 30 minutes later, $\mathrm{PYO}^{\mathrm{Sa}} \rightarrow \mathrm{AB}$. As controls, we also treated parallel cultures of $S$. aureus Newman with antibiotics only, $A B$, and with phage alone. In Fig. 2 (bacteriostatic antibiotics) and Fig. 3 (bacteriocidal antibiotics) we 
present $\mathrm{N}(24) / \mathrm{N}(0)$ ratios for the bacteria and phage for three independent experiments with each antibiotic and phage combination.

With the bacteriostatic antibiotics, tetracycline (TET), azithromycin (AZM), and linezolid (LZD), the greatest decline in the density of bacteria and increase in the density of phage obtained in the experiment where phage are used alone. There is clearly a negative synergy between these bacteriostatic antibiotics and the phage. Whether administered simultaneously or sequentially, at the concentrations used, these antibiotics prevent PYOSa from killing S. aureus Newman. When the phage are administered simultaneously with azithromycin and linezolid, the phage density declines.
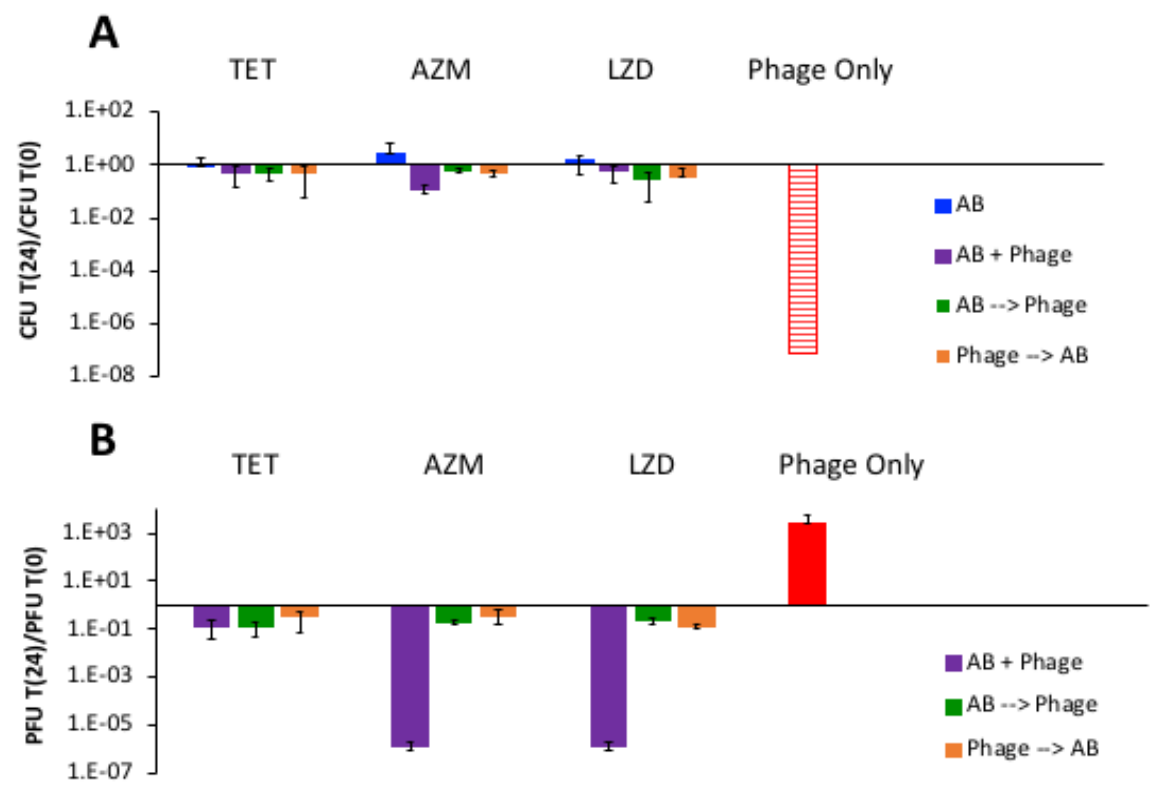

Fig. 2. Joint action of bacteriostatic antibiotics and PYOSa. The concentrations of these antibiotics are $10 \mu \mathrm{g} / \mathrm{ml}$. A. The ratio of the change in density of S. aureus after 24 hours of exposure to antibiotics (blue), antibiotics plus phage (purple, green, orange, refers to order of addition as explained in the text) or phage alone (red). Hash red, the density of $S$. aureus recovered was below the detection limit, $\sim 10^{2}$ cells per ml. B. The ratio of the change in the density of PYOSa after 24 hours of confronting wild-type $S$. aureus in combination with antibiotics or alone.

In Fig. 3, we present the results of experiments with bactericidal antibiotics, rifampin (RIF), oxacillin (OXA), ciprofloxacin (CIP), vancomycin (VAN), daptomycin (DAP) and kanamycin (KAN). The data suggest that the simultaneous or sequential administration of $\mathrm{PYO}^{\mathrm{Sa}}$ may modestly increase the rate at which bacteriocidal antibiotics kill $S$. aureus at the concentrations employed. However, as was observed for the parallel experiment with the bacteriostatic drugs (Fig. 2), the phage kill more $S$. aureus in the absence of antibiotics than with these drugs, an antagonistic interaction once again. The failure of rifampin to reduce the viable density of $S$. 
aureus can be attributed to rifampin resistance emerging. It should be noted however, that in one of the three treatments where PYO ${ }^{\text {Sa }}$ was used before adding rifampin, it prevented the ascent of resistance. Most interestingly, while treatment with rifampin, oxacillin, and ciprofloxacin allowed PYOSa to replicate, this was not the case for vancomycin, daptomycin, and kanamycin, which appeared to suppress the replication of PYOSa.

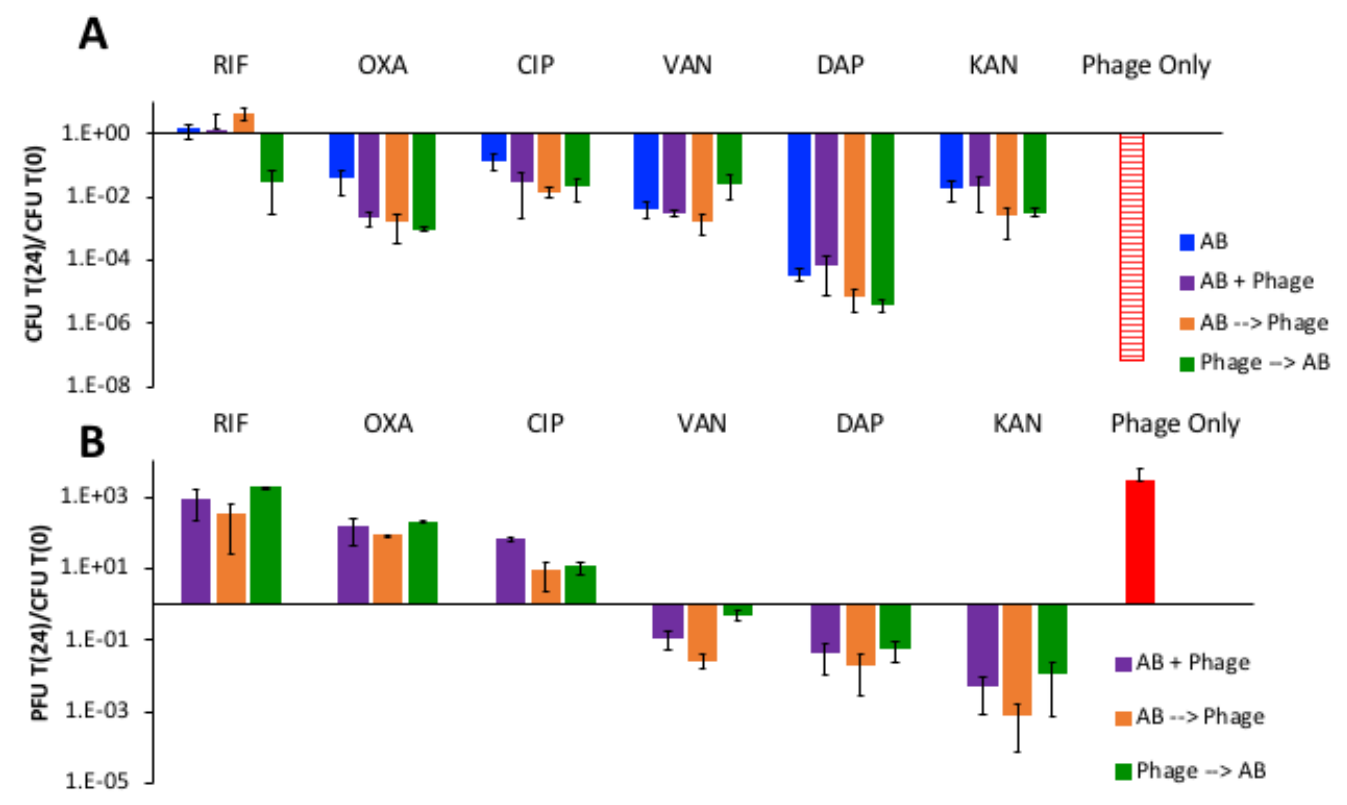

Fig. 3. The joint action of bactericidal antibiotics and PYOSa. Concentrations of the different antibiotics in $\mu \mathrm{g} / \mathrm{ml}$ (RIF 0.02, OXA 3, CIP 0.5, VAN 8, DAP 64, KAN 46), corresponding to minimum bacteriocidal concentrations. A. The ratio of the change in density of $S$. aureus after 24 hours of exposure to antibiotics, antibiotics and phage, or phage alone. Hash red, the density of $S$. aureus recovered was below the detection limit, $\sim 10^{2}$ per $\mathrm{ml}$. B. The ratio of the change in the density of PYO $\mathrm{O}^{\mathrm{Sa}}$ after 24 hours of confronting $S$. aureus Newman in combination with antibiotics or alone.

(9) Sequential treatment, phage followed by antibiotics: The serial transfer results presented in Fig. $1 B$ indicate that as a consequence of the emergence and ascent of small colony variants, $\mathrm{PYO}^{\mathrm{Sa}}$ by itself will not be able to control an $S$. aureus population for an extended time. Although the phage continue to be present, the density of the bacteria returns to levels similar to that of the phage-free control (Fig. $1 B$ ). We asked what result would be obtained if antibiotics were administered to these populations? To address this question, we performed the serial transfer experiments with the addition of bactericidal antibiotics following the phagemediated reduction in the density of wild-type $S$. aureus observed during the first 24 hours. The results of these experiments are presented in Fig. 4. 

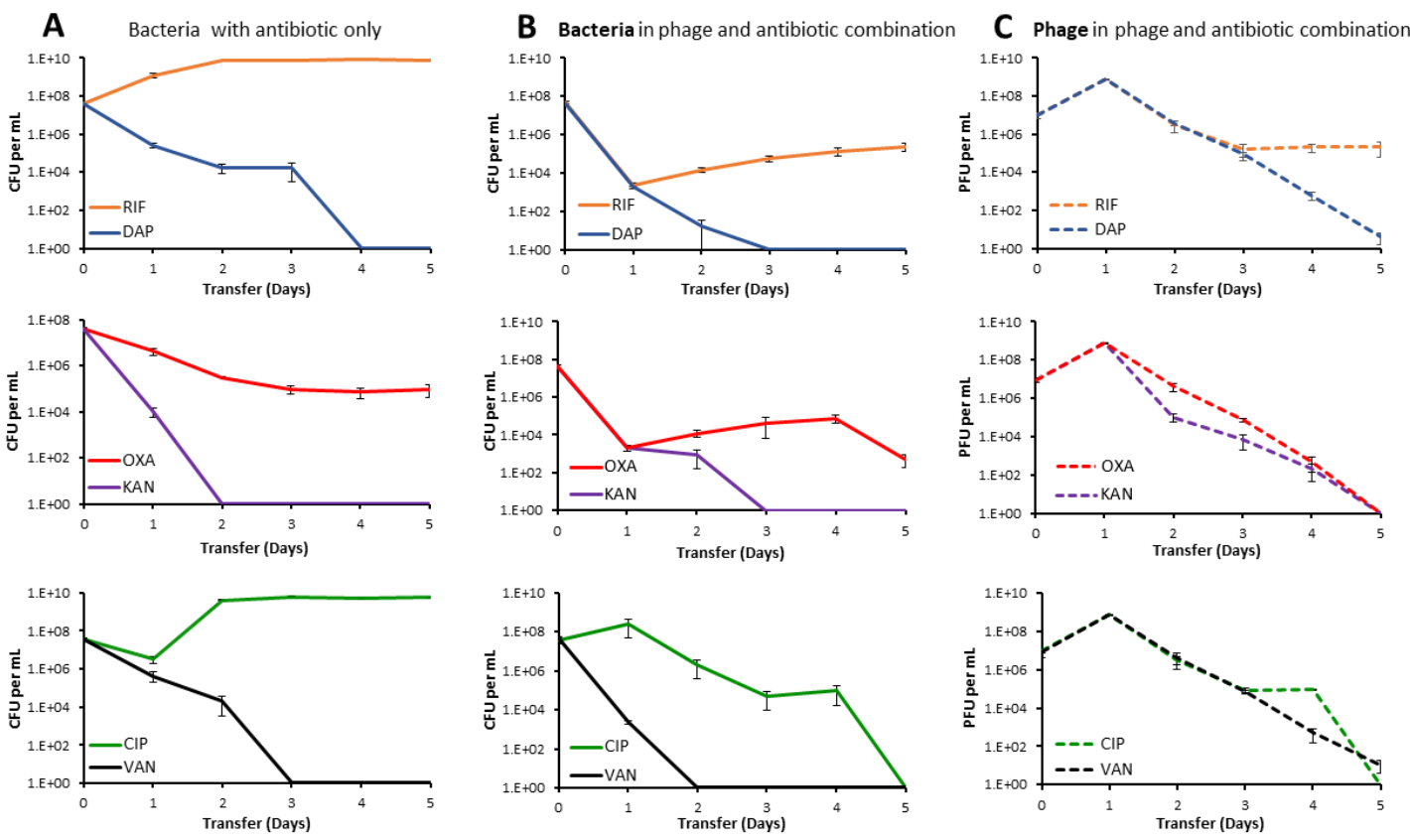

Fig. 4. Changes in the densities of bacteria and phage in serial transfer cultures treated with antibiotics alone, or in combination with phage. Column A. Bacteria treated with antibiotics alone. Column B. Bacterial densities in cultures containing both the antibiotic and the phage. Column C. Phage densities in cultures containing both the antibiotic and the phage. Mean and standard errors of 3 independent experiments. Row 1. Treatment with RIF and DAP, 0.02 and $64 \mu \mathrm{g} / \mathrm{ml}$, respectively. Row 2 . Treatment with $\mathrm{KAN}$ and $\mathrm{OXA}, 46$ and $3 \mu \mathrm{g} / \mathrm{ml}$ respectively, Row 3. Treatment with CIP and VAN, 0.5 and $8 \mu \mathrm{g} / \mathrm{ml}$ respectively.

Three of the antibiotics, DAP, KAN and VAN, were alone sufficient to eliminate the $S$. aureus population (Fig. 4A). In contrast, with RIF by the second transfer the density of $S$. aureus in treated cultures reached a density observed for antibiotic and phage free cultures $\left(\sim 2 \times 10^{9}\right.$ bacterial per $\left.\mathrm{ml}\right)$. The reason for the failure of RIF to clear the culture was the emergence of mutants resistant to this drug. All six colonies tested from the $5^{\text {th }}$ transfer were resistant to RIF. However, the viable cells recovered from the phage and RIF combination cultures were as sensitive to RIF as the antibioticfree controls. Neither OXA nor CIP alone cleared the cultures. In the case of OXA, the viable cell density declined but continued to persist at a density of approximately $10^{5}$ cells per ml. The bacteria recovered from these cultures were sensitive to OXA. We postulate that this leveling off can be attributed to persistence (32); see SI Appendix, Section VI, Fig. S5. In the case of CIP, the initial exposure led to a substantial decline in the viable cell density of $S$. aureus, however, following the second transfer the bacterial population recovered and was sustained at densities similar to that in the antibiotic-free controls. The colonies of $S$. aureus recovered at the end of this experiment were susceptible to CIP. We postulate that these resurrection dynamics could be attributed to heteroresistance (33), see SI Appendix, Section VI, Fig S5. 


\section{Discussion and conclusions}

On first consideration, $\mathrm{PYO}^{\mathrm{Sa}}$ seems to be an ideal phage for treating $S$. aureus infections. The results of this study provide evidence in support of three virtues of $\mathrm{PYO}^{\mathrm{Sa}}$ as a therapeutic phage. 1) PYO ${ }^{\mathrm{Sa}}$ is likely to kill virtually all methicillinresistant as well as methicillin-sensitive $S$. aureus. 2) $S$. aureus are unable to generate classical surface-resistant mutants to PYO ${ }^{\mathrm{Sa}}$, thus, cocktails of multiple phages would not be needed to ensure coverage or prevent resistance. 3) $S$. aureus Newman has a high adsorption rate and burst size with PYO ${ }^{\text {Sa }}$ and, when first confronting growing populations of $S$. aureus, the bacteria are killed, and the phage replicate at a high rate.

On the downside, our experiments raise caveats about the use of PYO ${ }^{\text {Sa }}$ for treating $S$. aureus infections alone and suggest a possible liability as well. Not only is PYOSa unable to clear cultures of $S$. aureus Newman, it selects for potentially pathogenic small colony variants, (34-37) that are refractory to this phage. Through a "leaky resistance" mechanism (38), the phage continue to be maintained, and the bacterial population continues to persist at densities not much less than they do in the absence of PYOSa. Although not observed for the small colony variants tested here, at least some small colonies are more resistant to antibiotics than the bacteria from which they are derived $(27,39,40)$.

For phage therapy to be a practical and acceptable enterprise, these viruses would have to be used in combination with antibiotics. Our results indicate that when administered simultaneously, or nearly simultaneously with antibiotics, PYOSa does worse in killing $S$, aureus than it does alone PYOSa. The ribosome-targeting bacteriostatic antibiotics, tetracycline, azithromycin, and linezolid, suppress the ability of PYOSa to kill $S$. aureus Newman. This observation is consistent with the failure of $\mathrm{PYO}^{\text {Sa }}$ to replicate on stationary phase populations of $S$. aureus. It is also consistent with the Numbers Game hypothesis for the action of bacteriostatic antibiotics (41) according to which, the number of free ribosomes is too low to support the protein synthesis needed for replication of the phage.

When administered simultaneously with bactericidal antibiotics, $\mathrm{PYO}^{\mathrm{Sa}}$ is also less effective in killing $S$. aureus than it is in the absence of these drugs. We postulate that this can be attributed to pharmaco and population dynamics of the joint action of antibiotics and phage; the antibiotics reduce the densities of the bacteria substantially and thereby lower the capacity of the phage to replicate (SI Appendix, Section IV).

Sequential treatment with PYOSa and antibiotics: Our experiments suggest a way to deal with the major caveat and potential liability of treatment with PYO ${ }^{\mathrm{Sa}}$ alone, the recovery of the bacterial population due to the ascent of small colony variants. The administration of bactericidal antibiotics following the initial decline in the density of bacteria due to $\mathrm{PYO}^{\mathrm{Sa}}$ prevents bacterial population recovery and eliminates or 
prevents the selection of small colony variants. The latter is not the case when the antibiotics are used alone. One interpretation of this is that sequential treatment, initially with phage, then with a bactericidal antibiotic may be more effective than treatment with antibiotics alone or phage alone.

Conclusion and Recommendation: We interpret the results of this in silico and in vitro study to suggest that $\mathrm{PYO}^{\mathrm{Sa}}$ will be effective for treatment of $S$. aureus infections, but only if the administration of bactericidal antibiotics follows that of phage. The next step will, of course, be to test this sequential phage and antibiotic treatment hypothesis with $S$. aureus infections in experimental animals.

We suggest that the in vitro methods used to explore the potential efficacy of PYOSa would also be useful for evaluating other phages being developed for treating bacterial infections. Existing data suggested that PYOSa met all of the criteria desired for a phage to be effective for therapy; yet, the in vitro experiments performed here uncovered a limitation and potential liability of using this phage for therapy that would not have anticipated and they also subsequently revealed a way to deal with said limitation and liability.

\section{Materials and Methods}

\section{Strains and growth media}

Unless otherwise noted, all experiments were performed from derivatives of the parent strain $S$. aureus Newman (ATCC 25904). The parent $S$. aureus Newman was obtained from Bill Schafer of Emory University. The small colony variant and evolved strains were obtained from PYOSa challenged $S$. aureus Newman by experiments performed in our lab. The investigation for classical resistance was performed in the following MSSA strains obtained from Abraham Moller in the Reid Lab at Emory University: NRS52, NRS102, NRS180, NRS110, NRS252, NRS253, NRS266, NRS109, NRS148, and NRS205.

Bacterial cultures were grown at $37^{\circ} \mathrm{C}$ in Mueller-Hinter II (MHII) Broth [275710, $\mathrm{BD}^{\mathrm{TM}}$ ] and on Luria-Bertani Agar (LB) Plates [244510, BD ${ }^{\mathrm{TM}}$. PYO ${ }^{\text {Sa }}$ lysates were prepared from single plaques at $37^{\circ} \mathrm{C}$ in MHII broth alongside wild-type $S$. aureus Newman by plate lysis. Specifically, individual phage plaques were picked with a sterile stick, resuspended in $4 \mathrm{ml}$ of soft agar with $0.1 \mathrm{ml}$ of overnight bacterial culture and plated on top of phage plates. The plates were then incubated at $37^{\circ} \mathrm{C}$ overnight. The soft agar was scraped with a sterile iron scoop, resuspended in $10 \mathrm{ml}$ MHII with $\sim 0.5 \mathrm{ml}$ of chloroform to kill the surviving bacteria. The lysates were then centrifuged to remove the agar, sterilized by filtration $(0.2 \mu \mathrm{m})$ and stored at $4{ }^{\circ} \mathrm{C}$.

\section{Sampling bacterial and phage densities}


Bacteria and phage densities were estimated by serial dilutions in $0.85 \% \mathrm{NaCl}$ solution followed by plating. The total density of bacteria was estimated on LB (1.6\%) agar plates. To estimate the densities of free phage, chloroform was added to suspensions before serial dilutions. These suspensions were mixed with $0.1 \mathrm{~mL}$ of overnight MHII grown cultures of wild-type $S$. aureus Newman (about $5 \times 10^{8}$ cells per $\mathrm{mL}$ ) in $4 \mathrm{~mL}$ of LB soft (0.65\%) agar and poured onto semi-hard (1\%) LB agar plates.

\section{Parameter estimations}

The parameters critical for the interaction of the PYO ${ }^{\text {Sa }}$ phage and $S$. aureus Newman used in this study were estimated in independent experiments MHII broth. The maximum growth rate of different clones of $S$. aureus Newman was measured by Bioscreen, as described in (42). Phage burst sizes $(\beta)$ were estimated with one-step growth experiments similar to (43). Adsorption of $\mathrm{PYO}^{\text {Sa }}$ to $S$. aureus was estimated as described in (43).

\section{Serial transfer experiments}

All serial transfer experiments were carried out in $10 \mathrm{ml}$ MHII cultures grown at $37^{\circ} \mathrm{C}$ with vigorous shaking. The cultures were initiated by $1: 100$ dilution from $10-$ $\mathrm{mL}$ overnight cultures grown from single colonies. Phage was added to these cultures to reach the initial density of approximately $10^{6} \mathrm{PFU} / \mathrm{mL}$. At the end of each transfer, $0.1 \mathrm{~mL}$ of each culture was transferred into flasks with fresh medium (1:100 dilution). Simultaneously, $0.1 \mathrm{~mL}$ samples were taken for estimating the densities of colony-forming units (CFU) and plaque-forming units (PFU), by serial dilution and plating on solid agar.

\section{Antibiotics and their Sources}

Tetracycline, Oxacillin, Vancomycin, Kanamycin, Streptomycin (all from Sigma Aldrich), Azithromycin (Tocris), Daptomycin (MP Biochemicals), Rifampin (Applichem) and Linezolid (Chem- Impex International).

\section{Whole-genome sequencing}

For sequencing individual clones of $S$. aureus, genomic DNA was prepared using the MasterPure Gram-Positive kit, following the manufacturer's instructions (Epicentre, Illumina Inc., Madison, Wisconsin). Final DNA was resuspended in EB buffer.

Genomic DNA concentrations were measured in a Qubit 2.0 Fluorometer (Invitrogen via ThermoFisher Scientific). DNA was diluted to $0.2 \mathrm{ng} / \mu \mathrm{L}$ in water (Sigma-Aldrich, Sweden), and the samples were prepared for whole genome sequencing according to Nextera ${ }^{\circledR}$ XT DNA Library Preparation Guide (Illumina Inc., Madison, Wisconsin). After the PCR clean up-step, samples were validated for DNA fragment size distribution using the Agilent High Sensitivity D1000 ScreenTape System (Agilent Technologies, Santa Clara, California). Sequencing was performed using a MiSeq ${ }^{\mathrm{TM}}$ 
desktop sequencer, according to the manufacturer's instructions (Illumina Inc., Madison, Wisconsin). The sequencing data were aligned and analyzed in CLC Genomics Workbench version 11.0 (CLCbio, Qiagen, Denmark).

Acknowledgements: We thank Melony Ivey and Esther Lee for superb technical help. We are grateful to our Staphyloccus maven, Abraham (Jon) Moller, for providing strains and sage advice. And, to Waqas Chaudhry, Andrew Smith, and the reviewers of an earlier incarnation of this report for helpful comments and suggestions. Funds for this research were provided by grants from the US National Institutes of General Medical Sciences, R01 GM091875 and R35 GM 136407 (Bruce Levin) and Vetenskapsrådet (the Swedish Research Council) (2017-03593) and from the Scandinavian Society for Antimicrobial Chemotherapy (SLS-693211, SLS876451), Diarmaid Hughes. These funders had no role in study design, data collection and interpretation, or the decision to submit the work for publication.

\section{References}

1. F. D'Herelle, The Bacteriophage and Its Behavior (The Williams \& Wilkins Company, Baltimore, MD., 1926), pp. 578.

2. M. Ansaldi et al., "French Phage Network"-Third Meeting Report. Viruses 10 (2018).

3. G. Watts (2017) Phage therapy: revival of the bygone antimicrobial. (Elsevier).

4. K. E. Kortright, B. K. Chan, J. L. Koff, P. E. Turner, Phage Therapy: A Renewed Approach to Combat Antibiotic-Resistant Bacteria. Cell Host Microbe 25, 219232 (2019).

5. C. Schmidt, Phage therapy's latest makeover. Nat Biotechnol 37, 581-586 (2019).

6. B. Martel, S. Moineau, CRISPR-Cas: an efficient tool for genome engineering of virulent bacteriophages. Nucleic Acids Res 42, 9504-9513 (2014).

7. T. K. Lu, J. J. Collins, Engineered bacteriophage targeting gene networks as adjuvants for antibiotic therapy. Proc Natl Acad Sci U S A 106, 4629-4634 (2009).

8. V. N. Krylov, [Phagotherapy in terms of bacteriophage genetics: hopes, perspectives, safety, limitations]. Genetika 37, 869-887 (2001).

9. S. J. Labrie, J. E. Samson, S. Moineau, Bacteriophage resistance mechanisms. Nat Rev Microbiol 8, 317-327 (2010).

10. Z. Moradpour, A. Ghasemian, Modified phages: novel antimicrobial agents to combat infectious diseases. Biotechnol Adv 29, $732-738$ (2011).

11. R. J. Dubos, J. H. Straus, C. Pierce, The Multiplication of Bacteriophage in Vivo and Its Protective Effect against an Experimental Infection with Shigella Dysenteriae. J Exp Med 78, 161-168 (1943).

12. J. J. Bull, B. R. Levin, T. DeRouin, N. Walker, C. A. Bloch, Dynamics of success and failure in phage and antibiotic therapy in experimental infections. $B M C$ Microbiol 2, 35 (2002).

13. R. T. Schooley et al., Development and Use of Personalized Bacteriophage-Based Therapeutic Cocktails To Treat a Patient with a Disseminated Resistant Acinetobacter baumannii Infection. Antimicrob Agents Chemother 61 (2017). 
14. B. K. Chan et al., Phage treatment of an aortic graft infected with Pseudomonas aeruginosa. Evol Med Public Health 2018, 60-66 (2018).

15. R. M. Dedrick et al., Engineered bacteriophages for treatment of a patient with a disseminated drug-resistant Mycobacterium abscessus. Nat Med 25, 730-733 (2019).

16. G. Xia et al., Wall Teichoic Acid-Dependent Adsorption of Staphylococcal Siphovirus and Myovirus. Journal of Bacteriology 193, 4006-4009 (2011).

17. T. Suzuki, J. G. Swoboda, J. Campbell, S. Walker, M. S. Gilmore, In vitro antimicrobial activity of wall teichoic acid biosynthesis inhibitors against Staphylococcus aureus isolates. Antimicrob Agents Chemother 55, 767-774 (2011).

18. G. Xia, T. Kohler, A. Peschel, The wall teichoic acid and lipoteichoic acid polymers of Staphylococcus aureus. International Journal of Medical Microbiology 300, 148-154 (2010).

19. S. McCallin, S. A. Sarker, S. Sultana, F. Oechslin, H. Brussow, Metagenome analysis of Russian and Georgian Pyophage cocktails and a placebo-controlled safety trial of single phage versus phage cocktail in healthy Staphylococcus aureus carriers. Environ Microbiol 20, 3278-3293 (2018).

20. S. O'Flaherty et al., Genome of Staphylococcal Phage K: a New Lineage of Myoviridae Infecting Gram-Positive Bacteria with a Low G+C Content. Journal of Bacteriology 186, 2862-2871 (2004).

21. Y. Sato'o et al., Tailor-made gene silencing of Staphylococcus aureus clinical isolates by CRISPR interference. PLoS One 13, e0185987-e0185987 (2018).

22. S. E. Hsieh, H. H. Lo, S. T. Chen, M. C. Lee, Y. H. Tseng, Wide host range and strong lytic activity of Staphylococcus aureus lytic phage Stau2. Appl Environ Microbiol 77, 756-761 (2011).

23. E. Kutter et al., Phage therapy in clinical practice: treatment of human infections. Current pharmaceutical biotechnology 11, 69-86 (2010).

24. J. Dickey, V. Perrot, Adjunct phage treatment enhances the effectiveness of low antibiotic concentration against Staphylococcus aureus biofilms in vitro. PLoS One 14, e0209390 (2019).

25. P. D. Fey et al., A Genetic Resource for Rapid and Comprehensive Phenotype Screening of Nonessential <span class="named-content genus-species" $\mathrm{id}=$ "named-content-1" $>$ Staphylococcus aureus $</$ span $>$ Genes. mBio 4, e0053700512 (2013).

26. B. R. Levin, F. M. Stewart, L. Chao, Resource - limited growth, competition , and predation: a model and experimental studies with bacteria and bacteriophage. American Naturalist 977, 3-24 (1977).

27. S. Cao, D. L. Huseby, G. Brandis, D. Hughes, Alternative Evolutionary Pathways for Drug-Resistant Small Colony Variant Mutants in Staphylococcus aureus. mBio 8 (2017).

28. A. M. Stranden, K. Ehlert, H. Labischinski, B. Berger-Bachi, Cell wall monoglycine cross-bridges and methicillin hypersusceptibility in a fem AB null mutant of methicillin-resistant Staphylococcus aureus. J Bacteriol 179, 9-16 (1997). 
29. M. W. Climo, K. Ehlert, G. L. Archer, Mechanism and suppression of lysostaphin resistance in oxacillin-resistant Staphylococcus aureus. Antimicrob Agents Chemother 45, 1431-1437 (2001).

30. J. M. Monteiro et al., Peptidoglycan synthesis drives an FtsZ-treadmillingindependent step of cytokinesis. Nature 554, 528-532 (2018).

31. I. C. McCall, N. Shah, A. Govindan, F. Baquero, B. R. Levin, Antibiotic Killing of Diversely Generated Populations of Nonreplicating Bacteria. Antimicrob Agents Chemother 63 (2019).

32. N. Q. Balaban et al., Publisher Correction: Definitions and guidelines for research on antibiotic persistence. Nat Rev Microbiol 17, 460 (2019).

33. H. Nicoloff, K. Hjort, B. R. Levin, D. I. Andersson, The high prevalence of antibiotic heteroresistance in pathogenic bacteria is mainly caused by gene amplification. Nat Microbiol 4, 504-514 (2019).

34. B. Kahl et al., Persistent Infection with Small Colony Variant Strains of Staphylococcus aureus in Patients with Cystic Fibrosis. The Journal of Infectious Diseases 177, 1023-1029 (1998).

35. C. von Eiff et al., A site-directed Staphylococcus aureus hemB mutant is a smallcolony variant which persists intracellularly. Journal of Bacteriology 179, 47064712 (1997).

36. P. Sendi, R. A. Proctor, Staphylococcus aureus as an intracellular pathogen: the role of small colony variants. Trends in Microbiology 17, 54-58 (2009).

37. C. von Eiff, Staphylococcus aureus small colony variants: a challenge to microbiologists and clinicians. International Journal of Antimicrobial Agents 31, 507-510 (2008).

38. W. N. Chaudhry et al., Leaky Resistance and the conditions for the existience of lytic bacteriophage. PLoS Biology 16:e2005971 (2018).

39. N. Baumert et al., Physiology and Antibiotic Susceptibility of Staphylococcus aureus Small Colony Variants. Microbial Drug Resistance 8, 253-260 (2002).

40. T. Norstrom, J. Lannergard, D. Hughes, Genetic and phenotypic identification of fusidic acid-resistant mutants with the small-colony-variant phenotype in Staphylococcus aureus. Antimicrob Agents Chemother 51, 4438-4446 (2007).

41. B. R. Levin et al., A Numbers Game: Ribosome Densities, Bacterial Growth, and Antibiotic-Mediated Stasis and Death. MBio 8 (2017).

42. J. Concepción-Acevedo, H. N. Weiss, W. N. Chaudhry, B. R. Levin, Malthusian parameters as estimators of the fitness of microbes: A cautionary tale about the low side of high throughput. PLoS One 10, e0126915 (2015).

43. E. L. Ellis, M. Delbruck, The Growth of Bacteriophage. J Gen Physiol 22, 365384 (1939). 


\section{SUPPLEMENTARY INFORMATION for}

\section{Joint antibiotic and phage therapy: addressing the limitations of a seemingly ideal phage for treating Staphylococcus aureus infections}

Brandon A. Berryhill, Douglas L. Huseby, Ingrid C. McCall, Diarmaid Hughes, and Bruce R. Levin

\section{Host range}

In our report, we summarized the evidence that $\mathrm{PYO}^{\mathrm{Sa}}$, $\mathrm{s}$ host range included all 83 clinical isolates of Staphylococcus aureus tested. Much of those data are from LabCorp. The methods used are summarized in the following and the results obtained are summarized in Supplemental Table S1.

\section{$\underline{\text { Methods }}$}

The methods LabCorp employed, as detailed below, to test the sensitivity of these strains to $\mathrm{PYO}^{\mathrm{Sa}}$ were somewhat different than those we employed and presented in our report (See Results Section 1).

(i) The S. aureus strains tested were streaked for single colonies on TSB agar.

(ii) These single colonies were inoculated in $3 \mathrm{ml} \mathrm{LB}$ broth and grown overnight with shaking at $37^{\circ} \mathrm{C}$.

(iii) The overnight cultures were diluted by 1:30 in $3 \mathrm{ml} \mathrm{LB}$ broth and grown with shaking at $37^{\circ} \mathrm{C}$ to an OD $600 \mathrm{~nm}$ of between $0.3-1.0$.

(iv) $300 \mathrm{ul}$ of these cultures were added to $3 \mathrm{ml} \mathrm{LB}$ soft $(0.3 \%$ or $0.5 \%)$ agar supplemented with 3 ul $1 \mathrm{M} \mathrm{CaCl}_{2}$ and $3 \mathrm{ul} 1 \mathrm{M} \mathrm{MgCl}_{2}$ which were spread onto $\mathrm{LB}$ agar plates to form a lawn.

(v) $5 \mu 1$ of the $\mathrm{PYO}^{\mathrm{Sa}}$ lysates were spotted onto the lawns and incubated overnight.

(vi) Lysates were prepared from original spot plates and then re-spotted on the original spot plate host bacteria following the above conditions.

The Key to scoring the results of this assay follows:

\begin{tabular}{|c|c|}
\hline \multicolumn{2}{|c|}{ Key } \\
\hline- & No lysis \\
\hline$-/+$ & Weak lysis \\
\hline
\end{tabular}




\begin{tabular}{|c|c|}
\hline+ & Partial lysis (cloudy) \\
\hline++ & Complete lysis \\
\hline
\end{tabular}

Table S1 Assay for the susceptibility of $S$. aureus to phage $\mathrm{PYO}^{\mathrm{Sa}}$. Data provided by LabCorp.

\begin{tabular}{|c|c|c|c|}
\hline Species & Strain & Spot & Re-spot \\
\hline Staphylococcus aureus & 27660 & ++ & ++ \\
\hline Staphylococcus aureus & RN6390 & + & $-/+$ \\
\hline Staphylococcus aureus & $502 \mathrm{~A}$ & ++ & ++ \\
\hline Staphylococcus aureus & 6538 & ++ & ++ \\
\hline Staphylococcus aureus & 25923 & ++ & ++ \\
\hline Staphylococcus aureus subsp. aureus & 12600 & ++ & ++ \\
\hline Staphylococcus aureus & RN4220 & ++ & ++ \\
\hline Staphylococcus aureus subsp. aureus & BAA-1721 & ++ & ++ \\
\hline Staphylococcus aureus subsp. aureus & 14775 & ++ & ++ \\
\hline Staphylococcus aureus & AR0461 & ++ & ++ \\
\hline Methicillin-resistant S. aureus & $017-243$ & ++ & ++ \\
\hline Methicillin-resistant S. aureus & 041-332 & + & $-/+$ \\
\hline Methicillin-resistant S. aureus & 045-188 & ++ & ++ \\
\hline Methicillin-resistant S. aureus & $038-401$ & + & ++ \\
\hline Methicillin-resistant S. aureus & $049-841$ & ++ & ++ \\
\hline Methicillin-resistant S. aureus & USA300 & + & - \\
\hline Methicillin-resistant S. aureus & $002-639$ & + & + \\
\hline Methicillin-resistant S. aureus & $049-60$ & ++ & ++ \\
\hline Methicillin-resistant S. aureus & $026-485$ & ++ & ++ \\
\hline Methicillin-resistant S. aureus & 011-719 & ++ & ++ \\
\hline Methicillin-resistant S. aureus & $045-696$ & ++ & ++ \\
\hline Methicillin-resistant S. aureus & BAA-1707 & + & $-/+$ \\
\hline Methicillin-resistant S. aureus & BAA-1717 & + & + \\
\hline Methicillin-resistant S. aureus & BAA-1720 & ++ & ++ \\
\hline Methicillin-resistant S. aureus & BAA-1747 & + & - \\
\hline Methicillin-resistant S. aureus & BAA-1754 & ++ & + \\
\hline
\end{tabular}




\begin{tabular}{|c|c|c|c|}
\hline Methicillin-resistant S. aureus & BAA-1761 & ++ & ++ \\
\hline Methicillin-resistant S. aureus & BAA-1763 & ++ & ++ \\
\hline Species & Strain & Spot & Re-spot \\
\hline Methicillin-resistant S. aureus & BAA-1764 & ++ & $-1+$ \\
\hline Methicillin-resistant S. aureus & BAA-1766 & ++ & + \\
\hline Methicillin-resistant S. aureus & BAA-1768 & ++ & ++ \\
\hline Methicillin-resistant S. aureus & BAA-41 & ++ & ++ \\
\hline Methicillin-resistant S. aureus & BAA-42 & ++ & $-/+$ \\
\hline Multidrug-resistant resistant S. aureus & BAA-44 & ++ & + \\
\hline Methicillin-resistant S. aureus & BAA-1683 & ++ & + \\
\hline Methicillin-resistant S. aureus & BAA-2094 & ++ & ++ \\
\hline Methicillin-resistant S. aureus & BAA-2313 & ++ & ++ \\
\hline Methicillin-resistant S. aureus & 33592 & ++ & + \\
\hline Methicillin-resistant S. epidermis & 700583 & $-/+$ & - \\
\hline Methicillin-resistant S. haemolyticus & 700564 & $-/+$ & - \\
\hline Staphylococcus aureus subsp. aureus & BAA-1718 & ++ & ++ \\
\hline Methicillin-resistant S. aureus & AR0462 & ++ & - \\
\hline Methicillin-resistant S. aureus & AR0463 & ++ & ++ \\
\hline Methicillin-resistant S. aureus & AR0464 & $-/+$ & $-/+$ \\
\hline Methicillin-resistant S. aureus & AR0465 & ++ & + \\
\hline Methicillin-resistant S. aureus & AR0466 & + & - \\
\hline Methicillin-resistant S. aureus & AR0467 & ++ & + \\
\hline Methicillin-resistant S. aureus & AR0468 & ++ & + \\
\hline Methicillin-resistant S. aureus & AR0469 & ++ & + \\
\hline Methicillin-resistant S. aureus & AR0470 & ++ & + \\
\hline Methicillin-resistant S. aureus & AR0471 & ++ & - \\
\hline Methicillin-resistant S. aureus & AR0472 & ++ & ++ \\
\hline Methicillin-resistant S. aureus & AR0473 & ++ & ++ \\
\hline Methicillin-resistant S. aureus & AR0474 & ++ & + \\
\hline Methicillin-resistant S. aureus & AR0475 & + & - \\
\hline Methicillin-resistant S. aureus & AR0476 & ++ & ++ \\
\hline Methicillin-resistant S. aureus & AR0477 & ++ & ++ \\
\hline
\end{tabular}




\begin{tabular}{|r|r|r|r|}
\hline Methicillin-resistant S. aureus & AR0478 & ++ & ++ \\
\hline Methicillin-resistant S. aureus & AR0479 & ++ & ++ \\
\hline Species & Strain & Spot & Re-spot \\
\hline Methicillin-resistant S. aureus & AR0480 & + & + \\
\hline Methicillin-resistant S. aureus & AR0481 & ++ & ++ \\
\hline Methicillin-resistant S. aureus & AR0482 & ++ & ++ \\
\hline Methicillin-resistant S. aureus & AR0483 & ++ & ++ \\
\hline Methicillin-resistant S. aureus & AR0484 & ++ & ++ \\
\hline Methicillin-resistant S. aureus & AR0485 & ++ & ++ \\
\hline Methicillin-resistant S. aureus & AR0486 & ++ & ++ \\
\hline Methicillin-resistant S. aureus & AR0487 & + & $-/+$ \\
\hline Methicillin-resistant S. aureus & AR0488 & + & $-/+$ \\
\hline Methicillin-resistant S. aureus & AR0489 & + & - \\
\hline Methicillin-resistant S. aureus & AR0490 & ++ & ++ \\
\hline Methicillin-resistant S. aureus & AR0491 & $-/+$ & - \\
\hline Methicillin-resistant S. aureus & AR0492 & + & - \\
\hline
\end{tabular}

\section{Variation in the demise - resurrection population dynamics presented in Figure 1 B.}

To explore the generality of the $\mathrm{PYO}^{\mathrm{Sa}}-S$. aureus Newman serial transfer results presented in Figure 1B, we performed the experiments with ten $2 \mathrm{ml}$ and six $10 \mathrm{ml}$ cultures. Both had effectively the same initial densities of phage and bacteria. The dynamics of only one of the ten $2 \mathrm{ml}$ samples serially transferred were similar to the demise-resurrection dynamics observed from Figure 1B. While all six $10 \mathrm{ml}$ serial transfer cultures were turbid by the $5^{\text {th }}$ transfer, some did not become turbid until the $4^{\text {th }}$ transfer. We interpret this observation to be consistent with the hypothesis that the resurrection of these phage-exposed bacteria is a stochastic process in S. aureus and thus is more likely to occur in the $10 \mathrm{ml}$ populations because the total number of bacteria is 5fold greater than that in $2 \mathrm{ml}$ cultures.

Also consistent with this stochastic hypothesis is that the time before the recovery of the S. aureus population due to the ascent of small colony variants, S, is variable. In Figure 1B, all three serial transfer populations started the recovery to high density by the second transfer. This was not the case for a repeat of this experiment. The recovery of one of the three populations (noted in red) started at the end of the first transfer (Figure S1A).

In Figure 1D, where the serial transfer cultures were initiated with S. aureus isolated at the end of serial transfer experiments with the $\mathrm{PYO}^{\mathrm{Sa}}$, the evolved bacteria, the phage 
were maintained in one of three cultures and were lost in two. In the repeat of this experiment with three independently evolved strains, the phage were lost in all three (Figure S1B).
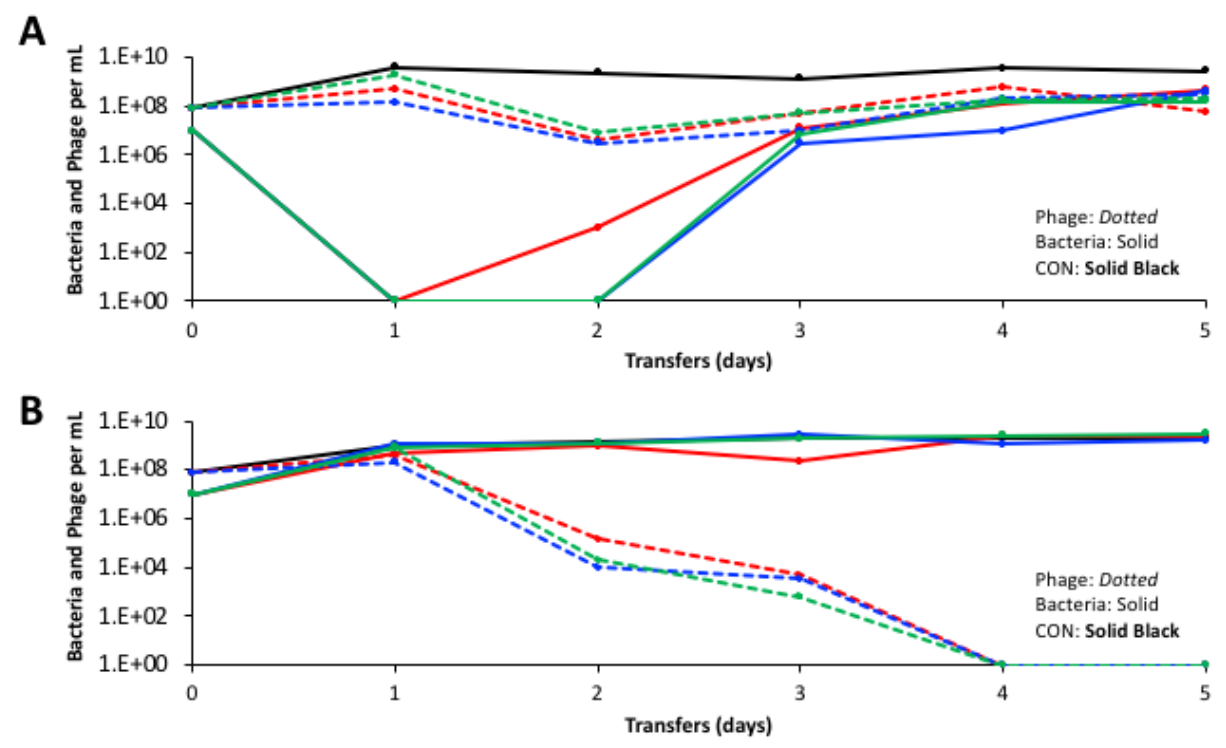

Figure S1 Changes in the densities of bacteria and phage in (1/100) $10 \mathrm{ml}$ serial transfer cultures of $S$. aureus Newman and $\mathrm{PYO}^{\mathrm{Sa}}$ and a phage-free control, CON. A) Three independent cultures initiated with $S$. aureus Newman (W) that had not previously been exposed to $\mathrm{PYO}^{\mathrm{Sa}}$. B) Three independent cultures initiated with $S$. aureus Newman (E) obtained from the $5^{\text {th }}$ transfer of independent serial passage experiments with $S$. aureus Newman mixed with $\mathrm{PYO}^{\mathrm{Sa}}$. 


\section{Genotypes and phenotypes of S. aureus mutants selected in the presence of PYO $^{\text {Sa }}$}

Table S2. Small colony variant $(\mathrm{S})$ mutants selected by exposure to $\mathrm{PYO}^{\mathrm{Sa}}$ in experiments of the type shown in Fig. $1 B$.

\begin{tabular}{|l|l|}
\hline Gene & Mutation \\
\hline fem $A$ & $\mathrm{G}>\mathrm{T}(\mathrm{nt}-\mathrm{g})$ \\
\hline fem $A$ & $\mathrm{G}>\mathrm{A}(\mathrm{nt}-12)$ \\
\hline fem $A$ & $\mathrm{M} 1 \mathrm{l}$ \\
\hline fem $A$ & $\Delta \mathrm{G}_{124}-\mathrm{A}_{12} 6$ \\
\hline fem $A$ & $\mathrm{I}_{171 \mathrm{AN}}$ \\
\hline fem $A$ & $\mathrm{Y}_{327} \mathrm{D}$ \\
\hline fem $A$ & 72 bp Dup $(\mathrm{nt} 987-1058)$ \\
\hline fem $A$ & $\mathrm{G} 331 \mathrm{D}$ \\
\hline fem $A$ & $\mathrm{G}_{368 \mathrm{~V}}$ \\
\hline
\end{tabular}

Mutations in $f e m A$ were identified after whole genome sequencing of small colony mutants.

Table S3. Genotypes of evolved (E) mutants from 5 different small colony variant (S) mutants.

\begin{tabular}{|c|c|c|}
\hline Primary Mutation & Compensatory mutation(s) & Annotated function of compensatory mutation(s) \\
\hline femA nt(-g) G>T & $m g r A W_{4} 8^{*}$ & HTH-type transcriptional regulator \\
\hline femA Metılle & $\operatorname{csh} A \triangle 258 b p$ & DEAD-box ATP-dependent RNA helicase \\
\hline femA Met1lle & $m g r A \Delta$ nt $160-216$ & HTH-type transcriptional regulator \\
\hline femA Met1lle & $\operatorname{rps} L \mathrm{G}_{126 \mathrm{D}}$ & $30 S$ ribosomal protein \\
\hline femA Tyr327Asp & clpX G177fs & ATP-dependent Clp protease \\
\hline femA Tyr327Asp & $\mathrm{CNH}_{3} 6 \_00075 \mathrm{H}_{32} 6 \mathrm{O}$ & Cyclic-di-AMP phosphodiesterase \\
\hline femA Tyr327Asp & $\mathrm{CNH}_{3} 6 \_06865 \Delta \mathrm{nt} 1011-105^{8}$ & 2-oxoacid:acceptor oxidoreductase subunit alpha \\
\hline femA Tyr327Asp & $\mathrm{CNH}_{3} 6{ }_{12385} \mathrm{~W}_{117} \mathrm{~L}$ & Lysostaphin \\
\hline femA Tyr327Asp & dacA $\vee_{78} \mathrm{G}$ & Diadenylate cyclase \\
\hline femA Tyr327Asp & femA $\mathrm{Q}_{346 \mathrm{R}}$ & femA internal suppressor \\
\hline femA Tyr327Asp & lpdA E245* & Dihydrolipoyl dehydrogenase \\
\hline femA Tyr327Asp & $n r n A O_{303 *}$ & Bifunctional oligoribonuclease PAP phosphatase \\
\hline femA Tyr327Asp & $\operatorname{sarA~K} 72:: \mathrm{IS}_{1181}$ & Transcriptional regulator \\
\hline femA Tyr327Asp & sarA R84 $\mathrm{C}_{1} \mathrm{CNH}_{3} 6 \_04445 \mathrm{~N}_{3} \mathrm{~S}$ & Transcriptional regulator, uncharacterized protein \\
\hline femA Tyr327Asp & $\operatorname{sarA} Y_{51} *$ & Transcriptional regulator \\
\hline femA Gly331Asp & lipL V $94 \mathrm{fs}$ & Octanoyl-[GcvH]:protein N-octanoyltransferase \\
\hline femA Gly331Asp & sarA Inv (nt - 51 to +78$)$ & Transcriptional regulator \\
\hline femA Gly331Asp & sarA RgoK & Transcriptional regulator \\
\hline femA Gly $368 \mathrm{Val}$ & rpoD G77fs & RNA polymerase sigma factor \\
\hline
\end{tabular}

Secondary compensatory mutations associated with the evolved state were identified by whole genome sequencing. 
Table S4. Relative growth rates and MICs of wild-type (W), small colony variant (S), and evolved (E) compensated mutants.

\begin{tabular}{|c|c|c|c|c|}
\hline \multicolumn{2}{|c|}{ Genotype / Mutations } & \multirow{2}{*}{$\begin{array}{c}\text { Growth Rate } \\
\text { Relative }\end{array}$} & \multicolumn{2}{|c|}{$\mathrm{MIC} \mathrm{mg/L}$} \\
\hline Primary & Compensatory & & Lysostaphin & Oxacillin \\
\hline Wild-type & & 1 & $\leq 0.008$ & 2 \\
\hline femA M1l & & 0.53 & 1 & 0.25 \\
\hline femA $\left.\right|_{171} \mathrm{~N}$ & & 0.48 & 4 & 0.125 \\
\hline femA $Y_{327} \mathrm{D}$ & & 0.59 & $>4$ & 0.125 \\
\hline femA $Y_{327} \mathrm{D}$ & femA $\mathrm{Q}_{34} 6 \mathrm{R}$ & 0.76 & 0.125 & 0.063 \\
\hline femA $Y_{327} D$ & sarA K72::IS1181 & 0.93 & $>4$ & 0.125 \\
\hline femA G331D & & 0.53 & $>4$ & 0.063 \\
\hline femA G331D & sarA INV (nt -51-+78) & 0.94 & $>4$ & 0.125 \\
\hline
\end{tabular}

Doubling time for wild-type is $25.8 \mathrm{~min}$.

\section{A hypothesis to account for the population and evolution dynamics of the $S$. aureus and PYO $^{\mathrm{Sa}}$.}

In our article we postulated that the dynamics presented in Figure 1B can be attributed to the generation of small colonies from the wild-type $S$. aureus Newman and their ascent due to selection mediated by $\mathrm{PYO}^{\mathrm{Sa}}$ phage. While the $\mathrm{PYO}^{\text {Sa }}$ phage adsorb to these small colony variants, they do not kill them, and the infecting phage are lost. The phage are maintained because the small colony variants are of low fitness and continually produce normal or near normal colony variants, an "evolved" state, that can support the replication of the phage. These evolved cells are not stable and continually produce small colonies. This hypothesis is illustrated in Figure S2.

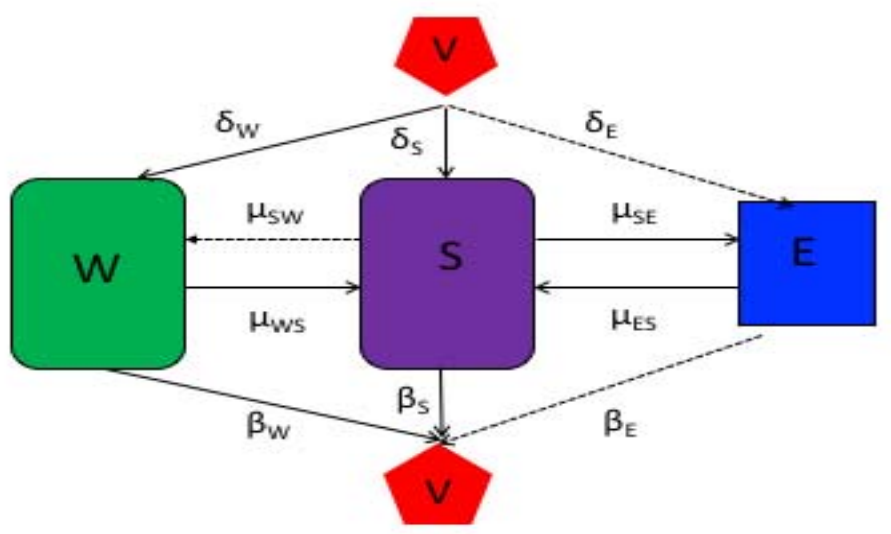


Figure S2 - A population dynamic model to account for the observed changes in the densities of bacteria and phage in Figure 1B. The variables, W, S and E are, respectively the wildtype $S$. aureus Newman, small colonies, and the evolved bacteria, cells per $\mathrm{ml}$, and $\mathrm{V}$ the PYOSa phage, particles per ml. The parameters $\delta_{\mathrm{W}}$, $\delta_{\mathrm{S}}$ and $\delta_{\mathrm{E}}$ are the adsorption rate constants per cell per ml. The parameters $\beta_{\mathrm{W}}, \beta_{\mathrm{S}}$ and $\beta_{\mathrm{E}}$ are the number of phage particles produced per infected cell burst. We assume that $\beta_{S}=0$, the $S$ are infected by PYO ${ }^{\text {Sa }}$ but the phage but do not replicate or kill these bacteria. The parameters $\mu_{\mathrm{SW}}, \mu_{\mathrm{WS}}, \mu_{\mathrm{SE}}$ and $\mu_{\mathrm{ES}}$ are the transition rates, per cell per hour, between the different states.

The population is in liquid culture in which there is a limiting resource of concentration, $r, \mu \mathrm{g} / \mathrm{ml}$. The net growth rate of the bacteria is proportional to its maximum rate of growth and a hyperbolic function of concentration of the limiting resource, $\mathrm{r}, \mu \mathrm{g} / \mathrm{ml}$ and a constant, $\mathrm{k}_{\mathrm{i}}$ which is the concentration of the resource when the growth rate is half its maximum value., where the subscript $\mathrm{i}$ is $\mathrm{W}, \mathrm{S}$ or $\mathrm{E}$ (1). We assume there is no latent period and upon adsorption, $\mathrm{W}$ and $\mathrm{E}$ are killed by the phage and instantly produce, $\beta_{W}$ and $\beta_{E}$ phage particles per cell (2). Although the phage adsorb to the small colonies, they are not killed. As the bacteria grow the concentration of the resource declines at a rate proportional to the net growth rates of the bacteria, and a conversion efficiency parameter, $e_{i} \mu \mathrm{g} / \mathrm{ml}$, the conversion efficiency, which is the amount of resource needed to produce a new cell of that type (3). To account for the declining physiological state of the bacteria as the bacteria approach stationary phase, $r=0$, we assume that the rates of phage adsorption and transitions between states is proportional to the net growth rate of that cell line. With these definitions and assumptions, the rates of change in the densities of the bacteria of different states, the density of free phage, and the concentration of the resource are given by the below set of coupled differential equations.

$$
\begin{aligned}
& \frac{d r}{d t}=-v_{W} \square \psi_{W}(r) \square e_{W} \square W-v_{S} \square \psi_{S}(r) \square e_{S} \square S-v_{E} \square \psi_{E}(r) \square e_{E} \square E \\
& \frac{d W}{d t}=v_{W} \square \psi_{W}(r) \square W-\delta_{W} \square W \square V \square \psi_{W}(r)+\mu_{S W} \square E \square \psi_{S}(r)-\mu_{W S} \square W \square \psi_{W}(r) \\
& \frac{d S}{d t}=v_{S} \square \psi_{S}(r) \square S+\mu_{W S} \square W \square \psi_{W}(r)-\mu_{S W} \square S \square \psi_{S}(r) \\
& \frac{d E}{d t}=v_{E} \square \psi_{S}(r) \square E-\delta_{E} \square E \square V \square \psi_{E}(r)+\mu_{S E} \square S \square \psi_{S}(r)-\mu_{E S} \square E \square \psi_{E}(r) \\
& \frac{d V}{d t}=\delta_{W} \square W \square V \square \psi_{W}(r) \square \beta_{W}+\delta_{E} \square E \square V \square \psi_{E}(r)-\delta_{S} \square V \square S \square \psi_{S} \square(r) \\
& \text { where } \psi_{W}(r)=\frac{r}{\left(r+k_{W}\right)}, \psi_{S}(r)=\frac{r}{\left(r+k_{S}\right)}, \text { and } \psi_{E}(r)=\frac{r}{\left(r+k_{E}\right)}
\end{aligned}
$$


Numerical Solutions - Simulations: To solve this set of coupled differential equations and those for the models that follow we use Berkeley Madonna. The population growth and phage infection parameters employed for these numerical solutions, simulations, are of the range estimated for $\mathrm{PYO}^{\mathrm{Sa}}$ and $S$. aureus Newman in MHII medium. To simulate a serial transfer mode of population maintenance every 24 hours there is a 100 -fold reduction in densities of the bacteria and phage and the resource concentration is restored to its maximum level of $1000 \mu \mathrm{g}$ per $\mathrm{ml}$. For copies of the program and instructions for it use write to blevin@emory.edu.

Simulation Results: In Figure S3, we follow the changes in the densities of the bacteria and phage in simulated serial populations. As observed in Figure 1B, the model predicts, as seen in Figure S3A, that upon the first encounter with the phage, V, the density of susceptible cells, W, and the total cell density of the bacteria, NT, will declines whilst that of the phage increase. In subsequent transfers, the population of bacteria recovers and becomes dominated by small colonies. As a consequence of the transitions between the different states of bacteria, the phage and all three bacterial populations are maintained with small colonies dominating the bacterial community. If we make the small colonies less efficient in the use of the resource, $\mathrm{e}_{\mathrm{c}}=5 \times 10^{-6}$ rather than $5 \times 10^{-7}$, the total density of the small colony population is lower (Figure S3A). When cultures of just small colonies are started without the phage, they are ultimately diluted out due to their lower growth rate and low production rate in the absence of the phage and are overtaken by the evolved population being generated through reversion from small colonies (Figure $\mathrm{S} 3 \mathrm{~B}$ ). When the evolved cells and phage are mixed, as observed in Figure 1D, the population recovers to full density more rapidly than when sensitive cells and phage are mixed (Figure S3C). However, in these simulations, the phage continue to be maintained, which was the case for only one of the two parallel experiments in the main body of the paper and in none of the parallel experiments presented in supplemental Figure S1B. This model can also account for why the mixture of the ancestral $S$. aureus Newman and small colony variants can maintain the phage (Figure 1E) with little effect on the density of bacteria (Figure S3D). 


\section{A}

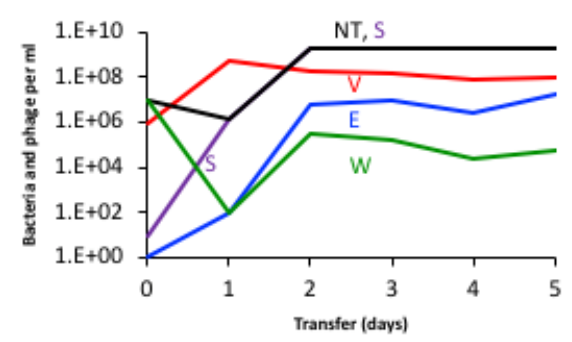

C

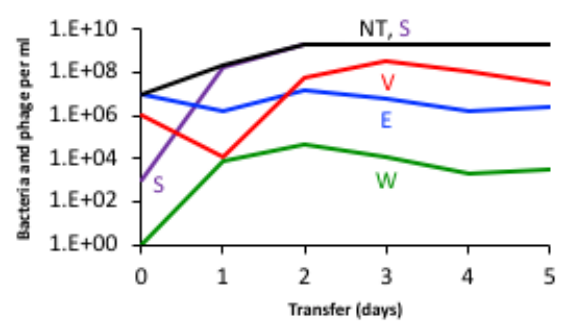

B

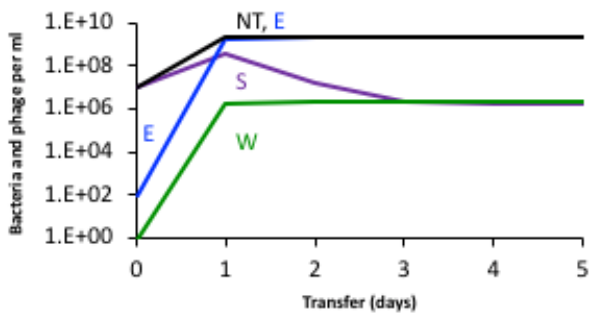

D

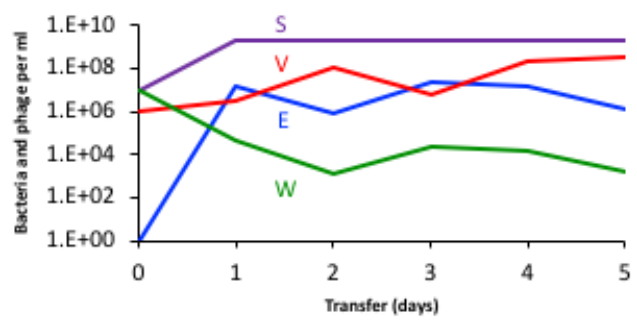

Figure S3 Simulated serial transfer populations. Changes in the densities of bacteria and phage in serial transfer culture. Standard parameters, $\mathrm{v}_{\mathrm{W}}=\mathrm{v}_{\mathrm{E}}=1.7, \mathrm{v}_{\mathrm{S}}=0.5, \delta_{\mathrm{W}}=\delta_{\mathrm{E}}=\delta_{\mathrm{S}}=$ $2 \times 10^{-7}, \beta_{\mathrm{W}}=\beta_{\mathrm{E}}=80, \beta_{\mathrm{S}}=0, \mathrm{e}_{\mathrm{w}}=\mathrm{e}_{\mathrm{e}}=5 \times 10^{-7}, \mathrm{ec}=5 \mathrm{Ex} 10^{-6}, \mathrm{C}=1000, \mathrm{k}_{\mathrm{W}}=\mathrm{k}_{\mathrm{E}}=1, \mathrm{k}_{\mathrm{S}}=10$, $\mu_{\mathrm{WS}}=10^{-6}, \mu_{\mathrm{SW}}=10^{-6}, \mu_{\mathrm{ES}}=10^{-3}, \mu_{\mathrm{SE}}=10^{-3}$. NT is the total density of bacteria. A) Serial transfer population with $\mathrm{W}$ and $\mathrm{V}$ and few $\mathrm{S}$ and $\mathrm{E}$. B) Serial transfer population initiated with $\mathrm{S}$ and miniority populations of $\mathrm{W}$ and $\mathrm{E}$, but no phage. C) Serial transfer population initiated with $\mathrm{E}$ and phage and minority populations of S and W. D) Serial transfer populations initiated with a mixture of $\mathrm{W}, \mathrm{S}$, and $\mathrm{V}$ and a minority of $\mathrm{E}$.

\section{V - The joint action of bactericidal antibiotics and phage.}

In our article, we postulate that the reason bactericidal antibiotics in combination with phage do worse than phage alone (Figure 4) can be attributed to the antibiotics reducing the density of bacteria and thereby the capacity of the phage to replicate. We illustrate this with the following model of the joint action of antibiotics and a bactericidal antibiotic.

Model of the joint action of antibiotics and phage: There are two populations of bacteria enumerated in cells per $\mathrm{ml}$. The populations are respectively $\mathrm{W}$ (wild-type, which are sensitive to antibiotics) and $\mathrm{P}$ (persisters which are sensitive to the phage but phenotypically resistant to antibiotics). There is a single antibiotic of concentration A in $\mu \mathrm{g} / \mathrm{ml}$, a lytic phage of density $\mathrm{V}$ particles per $\mathrm{ml}$, and a limiting resource $\mathrm{r}$ in $\mu \mathrm{g} / \mathrm{ml}$. The sensitive bacteria grow at a maximum rate, $\mathrm{v}_{\mathrm{N}}$, with the net growth/death rate $\psi(\mathrm{A}, \mathrm{r})$ being proportional to the concentration of the antibiotic and the limiting resource voila, 


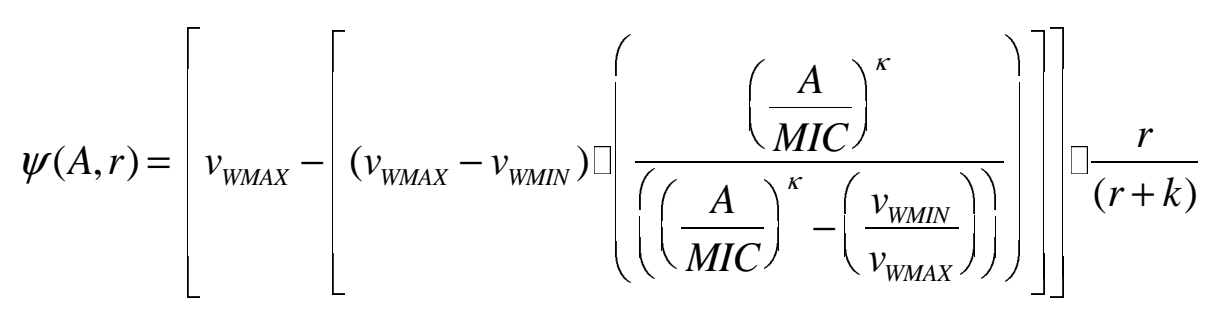

Where $\mathrm{V}_{\text {WMAX }}(>0)$ is the maximum growth rate, $\mathrm{v}_{\mathrm{WMIN}}(<0)$ is the minimum growth rate/maximum kill rate, MIC the minimum inhibitory concentration of the antibiotic, and a shape parameter, $\kappa$, such that the greater the value of $\kappa$ the more acute the function.

The persisters, $\mathrm{P}$, are non-replicating bacteria that are resistant to the antibiotic. The phage adsorb to the persisters but do not replicate on them. The rate constant of adsorption of the phage to sensitive cells is $\delta_{\mathrm{W}}$ and that to the persisters $\delta_{\mathrm{P}}$. Infections of phage to sensitive cells produce $\beta$ phage particles per cell. With a rate y per cell per hour, sensitive cells produce persisters, $\mathrm{W}-->\mathrm{P}$, and with a rate $\mathrm{x}$ per cell per hour, persisters produce sensitive cells, $\mathrm{P}-->\mathrm{W}$. With these definitions and assumptions, the rates of change in the densities of phage and the concentration of the resources are given by

$$
\begin{aligned}
& \frac{d r}{d t}=-\psi(A, r) \square W \square e-v_{M A X W} \square \psi(r) \square W \square e \\
& \frac{d W}{d t}=\psi(A, r) \square W-\delta_{W} \square W \square V \square \psi(r)-y \square W+x \square P \\
& \frac{d P}{d t}=y \square W-x \square P-\delta_{P} \square P \square V \square \psi(r) \\
& \frac{d V}{d t}=\delta_{W} \square V \square W \square \beta \square \psi(r)-\delta_{P} \square P \square V \square \psi(r)
\end{aligned}
$$

where $\psi(r)=\frac{r}{(r+k)}$ and $e \mu g$ is the amount of the limiting resource need to produce a new cell

Numerical Solutions - Simulations: To solve these equations and simulate the dynamics, we use Berkeley Madonna. Copies of the programs are available from blevin@emory.edu.

\section{$\underline{\text { Results }}$}

In Figure S4, we illustrate the rate of change in the total density of bacteria with a bactericidal antibiotic alone, with phage alone, and with the phage and antibiotic together. The rate of decline in the density of bacteria is lowest in the simulations where antibiotics are used alone. The highest rate of decline in the density of bacteria is obtained in the simulation where phage are used alone. When phage are used in combination with bactericidal antibiotics, they do not increase in density to the same extent that they do in the absence of the antibiotics. The leveling off in the density of bacteria is a consequence of persistence (4-6). 


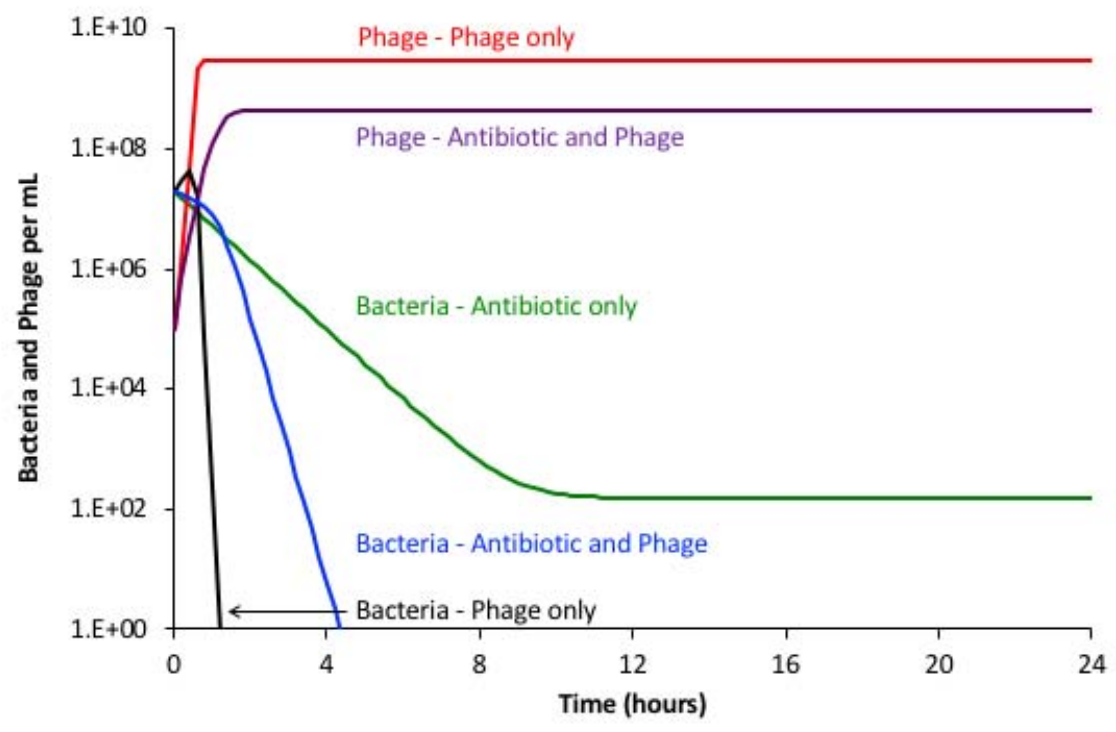

Figure S4 Simulation results: changes in the densities of bacteria and phage with different treatments. Parameter values. $\mathrm{v}_{\mathrm{SMAX}}=2.0, \mathrm{v}_{\mathrm{SMIN}}-2.0, \kappa=1.0, \mathrm{MIC}=1.0, \mathrm{e}=5 \times 10^{-7}$, $\mathrm{k}=1.0, \mathrm{x}=\mathrm{y}=10^{-5}, \delta_{\mathrm{P}}=10^{-8}, \delta_{\mathrm{S}}=10^{-8}, \beta=50, \mathrm{~A}=2 \mu \mathrm{g} / \mathrm{ml}, \mathrm{r}(0)=1000$.

\section{Persistence and heteroresistance}

In the absence of phage in serial transfer culture, the super MIC concentration of oxacillin does not eliminate the bacteria but rather maintains the density at levels markedly lower than the antibiotic-free controls (Figure 4 A center). We postulate that this can be attributed to persistence in serial transfer culture. In the absence of phage, ciprofloxacin initially reduces the density of $S$. aureus but in subsequent transfers, the density of these bacteria return to levels within the range anticipated for antibiotic-free controls (Figure 4A bottom). We postulated that these pharmacodynamics can be attributed to heteroresistance (7). In the following, we present the evidence for persistence for oxacillin-treated cultures and heteroresistance for ciprofloxacin-treated cultures. Using a mathematical - computer simulation model, we illustrate how these postulated mechanisms can account for the observed pharmacodynamics of these drugs with $S$. aureus Newman.

A model for antibiotics in serial transfer culture with persistence and heteroresistance: There are three populations of bacteria, wild-type, heteroresistant, and persisters with designations and densities, $\mathrm{W}, \mathrm{H}$, and $\mathrm{P}$ cells per $\mathrm{ml}$; a single antibiotic of concentration, $\mathrm{A} \mu \mathrm{g} / \mathrm{ml}$; and a limiting resource of concentration $\mathrm{r} \mu \mathrm{g} / \mathrm{ml}$. As in our model of the joint action of antibiotics and phage (II), we assume that the rate of growth, of bacteria of each of these states, $\psi_{\mathrm{i}}(\mathrm{A}, \mathrm{r})$ is a product of a Hill function for the antibiotic (4), and a Monod function for the resource (1). 
$\psi_{i}(A, r)=\left[v_{\text {iMAX }}-\left[\left(v_{i M A X}-v_{i M I N}\right)\left(\frac{\left(\frac{A}{M I C_{i}}\right)^{\kappa}}{\left(\left(\frac{A}{M I C_{i}}\right)^{\kappa}-\left(\frac{v_{i M I N}}{v_{i M A X}}\right)\right)}\right)\right]\right] \frac{r}{(r+k)}$

The subscript i represents the bacterial population, W, H, or P. For strain i, $v_{\text {imax }}(>0)$ is the maximum growth rate, $\mathrm{v}_{\mathrm{i} M I N}(<0)$ the minimum growth rate/maximum kill rate, and $\mathrm{MIC}_{\mathrm{i}}$ the minimum inhibitory concentration of the antibiotic. Bacteria of the same state have the same Hill coefficient and Monod constant, respectively $\kappa$ and $k$.

In these simulations, we assume the persisters can replicate, $\mathrm{V}_{\mathrm{PMAX}}>0$, and are resistant to the antibiotics, $\mathrm{V}_{\mathrm{PMIN}}=0$. As in (7), the MIC for the antibiotic of the heteroresistant cells is greater than the sensitive, $\mathrm{MIC}_{\mathrm{H}}>\mathrm{MIC}_{\mathrm{S}}$. There is a transition from $\mathrm{W}$ to $\mathrm{P}$ and from $\mathrm{P}$ to $\mathrm{W}$ respectively at rates, $\mathrm{x}$ and $\mathrm{y}$ per cell per hour, and a transition from $\mathrm{W}$ to $\mathrm{H}$ and $\mathrm{H}$ to $\mathrm{W}$ at rates, $\mathrm{x}_{\mathrm{H}}$, and $\mathrm{y}_{\mathrm{H}}$ per cell per hour. Finally, we assume that the concentration of the antibiotic can decline at a rate $d$ per hour. With these definitions and assumptions, the rates of change in the densities of the bacterial population and concentration of the antibiotic and resource are given by:

$$
\begin{aligned}
& \frac{d r}{d t}=-\psi(r) \square e \square\left(W \square v_{M A X W}+H \square v_{M A X H}\right) \\
& \frac{d W}{d t}=\psi_{W}(A, r) \square W-x \square W+y \square P-x_{H} \square W+y_{H} \square H \\
& \frac{d P}{d t}=v_{M A X P} \square \psi(r) \square P+x \square W-y \square P \\
& \frac{d H}{d t}=\psi_{H}(A, r) \square H+x_{H} \square W-y_{H} \square H \\
& \frac{d A}{d t}=-d \square A \\
& \text { where } \psi(r)=\frac{r}{(r+k)}
\end{aligned}
$$

Numerical Solutions: To solve these equations, we simulate the changes in the densities of the bacterial populations and changes in the concentration of the antibiotics with Berkeley Madonna. As in our experiments, in these simulations every 24 hours the density of the bacteria is reduced by a factor of 100 , and new resources and antibiotics are added. 

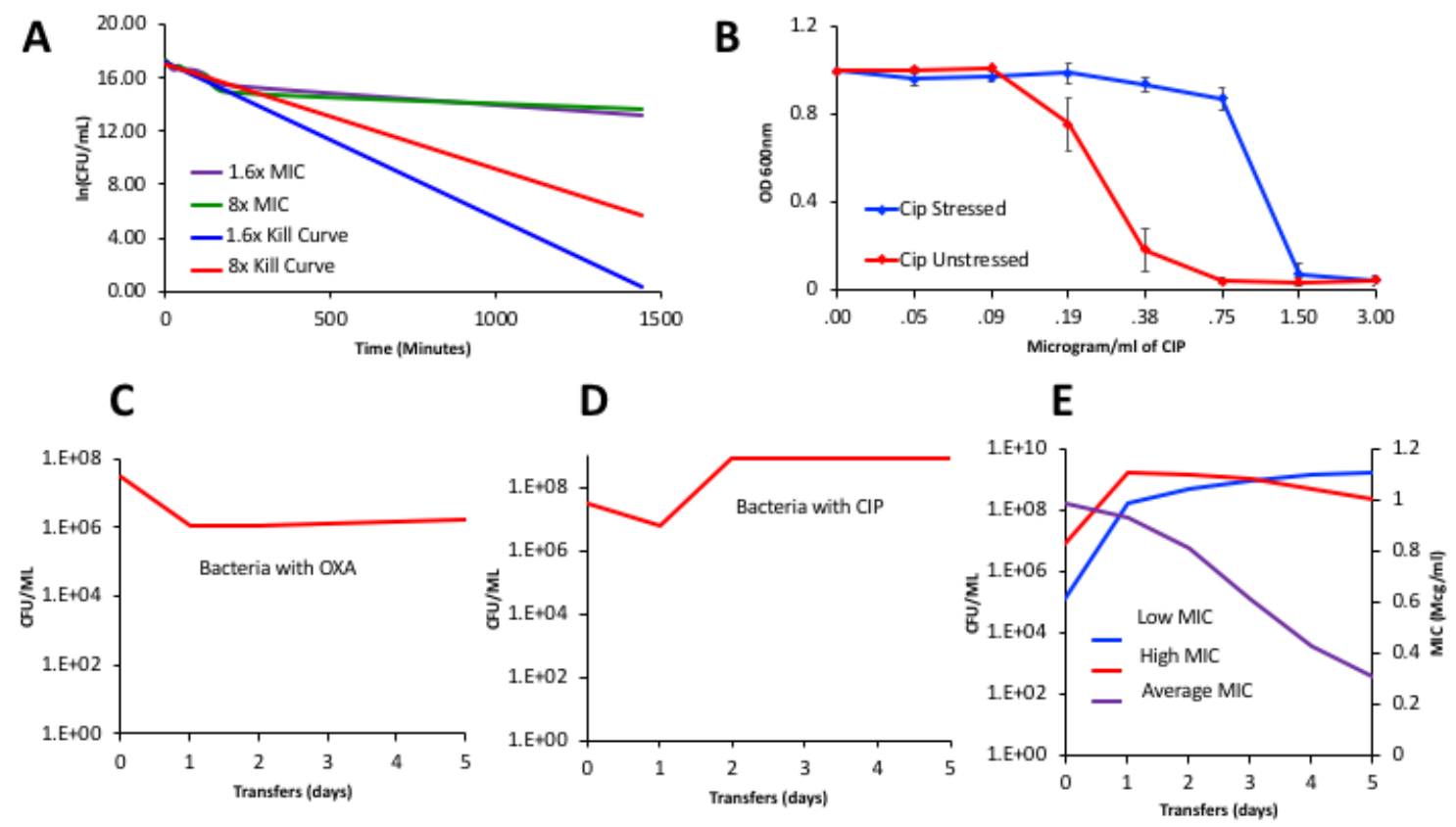

Figure S5 S. aureus antibiotic pharmacodynamics A) Natural log of the change in density of growing cultures of $S$. aureus Newman exposed to 1.6X and 8X MIC, low and high for 24 hours (1440 minutes). The green and purple lines are experimental changes in density estimated by plating; while the blue and red lines are the results of a linear regression of the changes in density estimated during the first 180 minutes of exposure. B) Changes in the optical density of experimental cultures of S. aureus Newman exposed to $5 \mu \mathrm{g} / \mathrm{ml}$ ciprofloxacin, mean and standard error of the ODs for three replicas. In red are cells that were treated with ciprofloxacin and then transferred without the presence of this drug for seven days before the MIC determination was performed. In blue are cells that were treated with ciprofloxacin for one week before the MIC was performed. C, D, E) Simulation Results Common parameters: $\mathrm{v}_{\mathrm{MAXS}}=1.5$, $\mathrm{v}_{\mathrm{MINS}}=-0.6, \mathrm{v}_{\mathrm{MAXSR}}=1.2, \mathrm{v}_{\mathrm{MINSR}}=-$ $3, \mathrm{ks}=1, \mathrm{kh}=1, \mathrm{k}=1, \mathrm{e}=5.0 \mathrm{E}-7 \mathrm{C})$ Changes in the densities of bacteria in serial transfer culture with persistence with parameters in the range estimated for $S$. aureus in oxacillin. Specific parameters: $\mathrm{vp}=0.2, \mathrm{MIC}_{\mathrm{S}}=1.8, \mathrm{x}_{\mathrm{P}}=1.0 \mathrm{e}-4, \mathrm{y}_{\mathrm{P}}=1 \mathrm{E}-3, \mathrm{~A}_{\mathrm{MAX}}=3$. Exposure to oxacillin does not start until the second transfer (compare to Figure 4A center). D) and E) Heteroresistance for ciprofloxacin $\mathrm{MIC}_{\mathrm{S}}=0.218, \mathrm{MIC}_{\mathrm{H}}=1, \mathrm{x}=1 \mathrm{E}-6, \mathrm{y}=1 \mathrm{e}-3, \mathrm{x}_{\mathrm{P}}=0$, $\mathrm{y}_{\mathrm{P}}=0, \mathrm{~A}_{\mathrm{MAX}}=0.5$ Changes in the densities of bacteria in serial transfer culture with heteroresistance with parameters in the range estimated for $S$. aureus in ciprofloxacin. Exposure to ciprofloxacin doesn't start until the second transfer (compare to Figure 4A bottom). E) Unique Parameters for $\mathrm{E}$ : $\mathrm{MIC}_{\mathrm{S}}=0.218, \mathrm{MIC}_{\mathrm{H}}=1, \mathrm{x}=1 \mathrm{E}-6,1 \mathrm{E}-3 \mathrm{x}=0, \mathrm{y}_{\mathrm{P}}=0$. Simulation of the changes in the densities of bacteria and average MIC of the antibiotic mixture of the low MIC sensitive and the high MIC heteroresistant populations in serial transfer culture in the absence of the antibiotics. 
In Figure S5A, we present the results of time-kill experiments with S. aureus Newman exposed to oxacillin. The observation that the rate of decline in the viable density of sensitive cells during the first few hours of exposure considerably exceeds the rate of decline later in the experiment is what would be anticipated for persistence. In Figure $\mathrm{S} 5 \mathrm{~B}$, we present the evidence for heteroresistance; higher concentrations of ciprofloxacin are needed to kill cultures initiated with bacteria exposed to ciprofloxacin than to kill bacteria cultured in the absence of this drug. If we allow for persistence with parameters in the range estimated for oxacillin the predicted changes in the density of $S$. aureus Newman in serial transfer culture with oxacillin are similar to those observed (compare Figures S4A and S4C). If we allow for heteroresistance with the parameter in the range estimated for $S$. aureus Newman in serial transfer culture with ciprofloxacin the results are similar to those observed (compare figures S5B and S5D). One property of heteroresistance is that when the bacteria are removed from the drug, the resistant population will decline in frequency, and the average MIC will decline to levels similar to that of the original sensitive population (7), see Figure S5E. 


\section{References}

1. J. Monod, The growth of bacterial cultures. Annual Review of Microbiology 3, 371-394 (1949).

2. B. R. Levin, F. M. Stewart, L. Chao, Resource - limited growth, competition , and predation: a model and experimental studies with bacteria and bacteriophage. American Naturalist 977, 3-24 (1977).

3. F. M. Stewart, B. R. Levin, Resource partitioning and the outcome of interspecific competition: a model and some general considerations. American Naturalist 107, 171-198 (1973).

4. R. R. Regoes et al., Pharmacodynamic functions: a multiparameter approach to the design of antibiotic treatment regimens. Antimicrob Agents Chemother 48, 3670-3676 (2004).

5. C. Wiuff et al., Phenotypic tolerance: antibiotic enrichment of noninherited resistance in bacterial populations. Antimicrob Agents Chemother 49, 1483-1494 (2005).

6. N. Q. Balaban et al., Definitions and guidelines for research on antibiotic persistence. Nat Rev Microbiol 17, 441-448 (2019).

7. H. Nicoloff, K. Hjort, B. R. Levin, D. I. Andersson, The high prevalence of antibiotic heteroresistance in pathogenic bacteria is mainly caused by gene amplification. Nat Microbiol 4, 504-514 (2019). 